TRANSACTIONS OF THE

AMERICAN MATHEMATICAL SOCIETY

Volume 358, Number 3 , Pages 1077-1103

S 0002-9947(05)03724-4

Article electronically published on April 22, 2005

\title{
MAYER BRACKETS AND SOLVABILITY OF PDES - II
}

\author{
BORIS KRUGLIKOV AND VALENTIN LYCHAGIN
}

\begin{abstract}
For the Spencer $\delta$-cohomologies of a symbolic system we construct a spectral sequence associated with a subspace. We calculate the sequence for the systems of Cohen-Macaulay type and obtain a reduction theorem, which facilitates computation of $\delta$-cohomologies by reducing dimension of the system. Using this algebraic result we prove an efficient compatibility criterion for a system of two scalar non-linear PDEs on a manifold of any dimension in terms of (generalized) Mayer brackets.
\end{abstract}

\section{INTRODUCTION}

In this paper we consider algebraic aspects of integrability of overdetermined PDEs systems $\mathcal{E} \subset J^{k}(\pi)$. The obstructions to formal integrability belong to the second Spencer $\delta$-cohomology groups $H^{i, 2}(\mathcal{E})[S$ ] and are called Weyl tensors $W_{l}$ Ly. Calculating these cohomologies (but not tensors, see details in the text) is a purely algebraic problem and can be posed for $H^{i, j}(g)$, where $g=\left\{g_{l} \subset\right.$ $\left.S^{l} T^{*} M \otimes \nu\right\}_{l \geq 0}$ is the system of symbols of a PDE $\mathcal{E}$ (manifold $M$ is the base of the bundle $\pi$ and $\nu$ is the fiber).

From an algebraic point of view a graded linear subspace $g \subset S T^{*} \otimes N$ is a symbolic system iff its $k$-dual $g^{*}$ (where $k=\mathbb{R}$ or $\mathbb{C}$ depending on the context) is an $S T$-module. We define $g$ to be a Cohen-Macaulay symbolic system if $g^{*}$ is a Cohen-Macaulay module.

Having a symbolic system $g=\left\{g_{l} \subset S^{l} T^{*} \otimes N\right\}$ and a subspace $V^{*} \subset T^{*}$ we define another system $\tilde{g}=\left\{g_{l} \cap S^{l} V^{*} \otimes N\right\} \subset S V^{*} \otimes N$. It is a symbolic system, which we call the $V^{*}$-reduction.

Theorem A. Let $g$ be a Cohen-Macaulay symbolic system, and let a subspace $V^{*} \subset$ $T^{*}$ be transversal to the characteristic variety of $g: \operatorname{codim}\left(\operatorname{Char}^{\mathbb{C}}(g) \cap P^{\mathbb{C}} V^{*}\right)=r=$ : codim $\operatorname{Char}^{\mathbb{C}}(g)$. For instance, this is so if $V^{*}$ is a non-characteristic subspace of dimension $r$. Then the Spencer cohomologies of the system $g$ and its $V^{*}$-reduction $\tilde{g}$ are isomorphic:

$$
H^{i, j}(g) \simeq H^{i, j}(\tilde{g})
$$

In some situations the considered formal reduction can be made into a genuine reduction. This happens, for example, if a Cohen-Macaulay system $\mathcal{E}$ is linear and has constant coefficients along $W=\operatorname{ann}\left(V^{*}\right)$. In fact, in this case the reduction is obtained via the Fourier transform along the $W$-variables.

Received by the editors December 16, 2002 and, in revised form, April 15, 2004.

2000 Mathematics Subject Classification. Primary 35N10, 58A20, 58H10, 35A30.

Key words and phrases. Mayer bracket, Spencer cohomology, Weyl tensor, integrals, characteristics, symbols, compatibility of PDEs.

(C)2005 American Mathematical Society Reverts to public domain 28 years from publication 
Corollary. If $\mathcal{E} \subset J^{k}(M)$ is a scalar system given by $r<n=\operatorname{dim}(M)$ equations and the characteristic variety $\operatorname{Char}^{\mathbb{C}}(g)$ has codimension $r$, the conclusion of Theorem $\mathrm{A}$ holds.

In fact, in this case the module $g^{*}=S T / I(g)$ is an algebra and it is CohenMacaulay whenever the characteristic ideal $I(g)$ is a complete intersection. We present other results on Spencer cohomologies in $\S \$ 2.2$ and 2.3

Remark 1. For first order PDEs our Theorem $\AA$ is somewhat complementary to Theorem A of Guillemin Gu. He considers a first order involutive system $g$, i.e., $H^{i, j}(g)=0$ for $j>0$, and proves that for any non-characteristic $V^{*}$ and $W=\operatorname{ann}\left(V^{*}\right) \subset T$ the image $g^{W}$ of the system $g$ in $S W^{*} \otimes N$ (called $W$-restriction) is involutive as well. Moreover, the symbols $g$ and $g^{W}$ are isomorphic. This implies that the only non-zero Spencer cohomologies are different: $H^{0, i}(g) \neq H^{0, i}\left(g^{W}\right)$.

In the considered case of Cohen-Macaulay system $g$ we have a different picture: the reduction remains Cohen-Macaulay, symbols $g$ and $\tilde{g}=g^{V}$ differ, but the $\delta$ cohomologies coincide (in terms of exact sequence (11), 22.1 in Guillemin's paper deals with the right-hand side, while we are concerned with the left).

We apply the reduction Theorem $\mathrm{A}$ to get a compatibility criterion for a system of two scalar PDEs. We obtained such a criterion via generalized Mayer brackets in the paper KL2 for the systems on a two-dimensional manifold. Here we generalize this result for an arbitrary base $M^{n}$.

Mayer bracket [,] for a pair of functions on the jet-space $J^{\infty}\left(\mathbb{R}^{n}\right)$ generalizes the classical Mayer bracket in the case of first order equations ([KL2]). Its restriction to the equation prolongation $[,]_{\mathcal{E}}$ is defined canonically, whence the bracket for functions on $J^{\infty}(M)$; see details in $\$ 3.2$. Note that for linear operators $F, G$ the bracket $[F, G]_{\mathcal{E}}$ is the usual (reduced) commutator.

Theorem B. Consider a system $\mathcal{E}$ of two scalar partial differential equations

$$
\begin{cases}F\left(x, u(x), \ldots, \frac{\partial^{|\sigma|} u}{\partial x^{\sigma}}\right)=0, & |\sigma| \leq k, \\ G\left(x, u(x), \ldots, \frac{\partial^{|\tau|} u}{\partial x^{\tau}}\right)=0, & |\tau| \leq l .\end{cases}
$$

Let the equations $F=0$ and $G=0$ have transversal characteristic varieties $\operatorname{Char}^{\mathbb{C}}(F)$, $\operatorname{Char}^{\mathbb{C}}(G) \subset P^{\mathbb{C}} T^{*} M$ and let at least one of them be without multiple components. Then the system $\mathcal{E}$ is formally integrable if and only if the Mayer bracket vanishes due to the system

$$
[F, G]_{\mathcal{E}}=0 .
$$

The absence of multiple components condition is redundant and is due to the involved machinery (the symbolic part of Theorem B - Proposition 12 - is an application of Theorem A). For second order equations this follows from [KL2, but the theorem holds true without the multiple components assumption always (the proof uses different ideas and will be published in a forthcoming paper).

Remark 2. Dimension of the symbol of the prolonged equation $\operatorname{dim} g_{s}$ is given by the Hilbert polynomial $p(s)$ of degree $\operatorname{dim} \operatorname{Char}(\mathcal{E})$. So this dimension tends to $\infty$ as $s \rightarrow \infty$ for $n=\operatorname{dim} M \geq 4$, stabilizes at a non-zero number $\operatorname{dim} g_{\infty}$ for $n=3$ and stabilizes at zero for $n=2$. So $n=2$ is the only case of finite type and for it we have smooth integrability ([KL2]). In the general case we can state only formal integrability or analytical one for analytic data (Cartan-Kähler theorem). 
The paper is organized as follows. In the first section we expose some relevant concepts from geometric theory of PDEs and rudiments of commutative algebra adapting them to the PDEs context. We refer to [S], KLV], Go, [KS], KL1] for details on the jet-theory and non-linear PDEs. In Section 2 we calculate Spencer cohomologies using spectral sequences and prove Theorem $\mathrm{A}$. Two proofs are given using the dualization and the theory of Cohen-Macaulay modules. Then in Section 3 we calculate the Weyl tensors for a system of two scalar PDEs and prove Theorem $B$. In the last section we give some physical and geometric applications of this theorem, exploiting a new concept of an auxiliary integral.

\section{Systems of PDEs}

1.1. Symbolic theory. Consider vector spaces $T$ of dimension $n$ and $N$ of dimension $m$ (usually over the field $\mathbb{R}$, but also possible over $\mathbb{C}$ ). The symmetric power $S T^{*}=\bigoplus_{i \geq 0} S^{i} T^{*}$ can be identified with the space of polynomials on $T$. Grading the de Rham complex of polynomial-valued forms

$$
0 \rightarrow S T^{*} \stackrel{d}{\rightarrow} S T^{*} \otimes T^{*} \stackrel{d}{\rightarrow} S T^{*} \otimes \Lambda^{2} T^{*} \rightarrow \cdots \stackrel{d}{\rightarrow} S T^{*} \otimes \Lambda^{n} T^{*} \rightarrow 0
$$

and tensorising it with $N$ one gets the Spencer $\delta$-complexes

$$
0 \rightarrow S^{k} T^{*} \otimes N \stackrel{\delta}{\rightarrow} S^{k-1} T^{*} \otimes N \otimes T^{*} \stackrel{\delta}{\rightarrow} \cdots \stackrel{\delta}{\rightarrow} S^{k-n} T^{*} \otimes N \otimes \Lambda^{n} T^{*} \rightarrow 0
$$

where $S^{i} T^{*}=0$ for $i<0$. By the Poincaré lemma the $\delta$-complex is exact.

For a linear subspace $h \subset S^{k} T^{*} \otimes N$ denote

$$
h^{(1)}=\left\{p \in S^{k+1} T^{*} \otimes N \mid \delta p \in h \otimes T^{*}\right\}
$$

and call it the first prolongation of $h$. Higher prolongations are defined inductively and satisfy $\left(h^{(k)}\right)^{(l)}=h^{(k+l)}$.

Definition 1. Define a symbolic system to be an infinite sequence of subspaces $g_{k} \subset S^{k} T^{*} \otimes N, k \geq 0$, with $g_{0}=S^{0} T^{*} \otimes N \simeq N$ (if $g_{0} \subset N$ is a strict inclusion one can shrink $N)$, that satisfy $g_{k} \subset g_{k-1}^{(1)}$.

With every such system we associate its Spencer $\delta$-complex of order $k$ :

$$
0 \rightarrow g_{k} \stackrel{\delta}{\rightarrow} g_{k-1} \otimes T^{*} \stackrel{\delta}{\rightarrow} g_{k-2} \otimes \Lambda^{2} T^{*} \rightarrow \cdots \stackrel{\delta}{\rightarrow} g_{k-n} \otimes \Lambda^{n} T^{*} \rightarrow 0 .
$$

The cohomology group at the term $g_{i} \otimes \Lambda^{j} T^{*}$ is denoted by $H^{i, j}(g)$.

Note that $g_{k}=S^{k} T^{*} \otimes N$ for $0 \leq k<r$ and the first number $r=r_{1}(g)$, where the equality is violated is called the minimal order of the system. Actually the system has several orders:

$$
\operatorname{ord}(g)=\left\{k \in \mathbb{Z}_{+} \mid g_{k} \neq g_{k-1}^{(1)}\right\} .
$$

Note that multiplicity of an order $m(r)=\operatorname{dim} g_{r-1}^{(1)} / g_{r}$ is equal to the dimension of the Spencer cohomology group $H^{r-1,1}(g)$. The Hilbert basis theorem (or Poincaré $\delta$-lemma) implies that the system of orders is always finite.

Definition 2. Call formal codimension of a symbolic system $g$ the number of elements in ord $(g)$ counted with multiplicities. In other words,

$$
\operatorname{codim}(g)=\sum_{k=1}^{\infty} \operatorname{dim} H^{k-1,1}(g) .
$$


In the previous paper KL2 we considered systems of formal codimension 2 and used the appropriate definition: From the whole set ord $(g)$ only the maximal and the minimal elements were considered as orders.

1.2. Commutative algebra approach. Denote by $\delta_{u}: S^{l+1} T^{*} \rightarrow S^{l} T^{*}$ the differentiation along $u \in T$. It is related to the Spencer differential and substitution via the formula $\delta_{u}=i_{u} \circ \delta$. We extend the differentiation to symmetric multi-vectors by setting $\delta_{v}=\delta_{u_{1}} \cdots \delta_{u_{k}}: S^{k+l} T^{*} \rightarrow S^{l} T^{*}$ on decomposable $v=u_{1} \cdots u_{k} \in S^{k} T$.

Let $g_{k} \subset S^{k} T^{*} \otimes N, k=0,1, \ldots$, be a system of subspaces. By the above we have the pairing $S^{k} T \otimes\left(S^{k+l} T^{*} \otimes N\right) \rightarrow S^{l} T^{*} \otimes N$ for $l \geq 0:(v, p) \mapsto \delta_{v} p$. Now via this pairing we define $I_{k}^{(l)}=\operatorname{ann} g_{k+l} \subset S^{k} T$ and $I^{(l)}=\bigoplus_{k} I_{k}^{(l)} \subset S T$.

Proposition 1. If $\left\{g_{k}\right\}$ is a symbolic system, then all $I^{(l)}$ are ideals in ST. For the scalar case $N=\mathbb{R}($ or $\mathbb{C})$ the reverse is true.

Proof. Let $g$ be a symbolic system. If $f \in I_{k}^{(l)}, u \in T$ and $\theta \in g_{k+l+1}$, then

$$
(f u) \theta=f(u \theta) \subset f\left(g_{k+l}\right)=\{0\} \Rightarrow f u \in I_{k+1}^{(l)} .
$$

For the scalar case with $I^{(0)}$ being an ideal the same formula shows that $g$ is a symbolic system.

Another way to deal with the system $g \stackrel{i}{\hookrightarrow} S T^{*} \otimes N$ is to consider its dual $g^{*}=\bigoplus g_{k}^{*}$, which is an epimorphic image of $S T \otimes N^{*}$ via the map $i^{*}$. The last space is naturally an $S T$-module and we can try to carry the module structure to $g^{*}$ by the formula $w \cdot i^{*}(v)=i^{*}(w \cdot v), w \in S T, v \in S T \otimes N^{*}$. Correctness of this operation has the following obvious meaning:

Proposition 2. System $g \subset S T^{*} \otimes N$ is symbolic iff $g^{*}$ is an $S T$-module.

From now on we suppose $g$ is a symbolic system. The dual $g^{*}$ is called the symbolic module, and its structure is given by

$$
(v \cdot \varkappa) p=\varkappa\left(\delta_{v} p\right), v \in S T, \varkappa \in g^{*}, p \in g .
$$

This module is Noetherian and the Spencer cohomology of $g$ defined in $\$ 1.1$ dualizes to the Koszul homology of $g^{*}$. This important fact will be used below.

Define the characteristic ideal by $I(g)=\operatorname{ann}\left(g^{*}\right) \subset S T$.

Proposition 3. The characteristic ideal satisfies $I(g)=\bigcap_{l=0}^{\infty} I^{(l)}$.

Note that due to the Noetherian property the intersection is actually finite.

Proof. The $k$-th graded component of the ideal $I(g)$ can be described via the pairing as $I_{k}=\left\{f \in S^{k} T \mid f \times g_{k+l} \rightarrow 0 \forall l \geq 0\right\}$. The statement follows.

Define the characteristic variety as the set of $v \in T^{*} \backslash\{0\}$ such that for every $k$ there exists a $w \in N \backslash\{0\}$ with $v^{k} \otimes w \in g_{k}$. This is a conical affine variety. We projectivize its complexification and denote the result by $\operatorname{Char}(g) \subset P^{\mathbb{C}} T^{*}$. Since only complex characteristics will be used, we omit the $\mathbb{C}$-superscript. Also for this variety we will denote by (co)dim its complex (co)dimension.

Another definition of characteristic variety is given via the characteristic ideal (the equivalence is proved in $[\underline{\mathrm{S}}$ ):

Proposition 4. Char $(g)=\left\{p \in P^{\mathbb{C}} T^{*} \mid f\left(p^{k}\right)=0 \forall f \in I_{k}, \forall k\right\}$. 
Let's look at this concept using the $A=S T$-module $g^{*}$. Recall (GR) its Chevalley dimension $\operatorname{dim}_{A} g^{*}$ is the minimal number $d$ of (homogeneous) elements $f_{1}, \ldots, f_{d} \in A$ such that the quotient $g^{*} /\left(f_{1}, \ldots, f_{d}\right) g^{*}$ is a finite-dimensional vector space. Thus due to equality $\operatorname{dim}_{A} g^{*}=\operatorname{dim}\left(A / \operatorname{ann}\left(g^{*}\right)\right)$, where $\operatorname{ann}\left(g^{*}\right)=I(g)$, we interpret $\operatorname{dim} g^{*}=\operatorname{dim} \operatorname{Char}(g)+1$ as the dimension of the affine characteristic variety.

A sequence of elements $f_{1}, \ldots, f_{s} \in A$ is called regular if $f_{i}$ is not a zero divisor in the $A$-module $g^{*} /\left(f_{1}, \ldots, f_{i-1}\right) g^{*}$. Depth (or cohomological codimension) depth $g^{*}$ of the module $g^{*}$ is the maximal number of elements in a regular sequence. The depth and dimension of a module $g^{*}$ are related by the following inequality: $\operatorname{depth} g^{*} \leq \operatorname{dim} g^{*}$. Now $g^{*}$ is called a Cohen-Macaulay module if $\operatorname{dim} g^{*}=\operatorname{depth} g^{*}$ (we'll omit sometimes the subscript $A$ ).

Proposition 5. An A-module $g^{*}$ is Cohen-Macaulay iff it is a free $B=S W$-module for some $W \subset T$ of dimension $\operatorname{dim}_{A} g^{*}$.

Proof. Due to the Noether normalization theorem there exists a finite homomorphism $B=S W \rightarrow A / I(g)$, where $W \subset T$ has dimension $\operatorname{dim}_{A} g^{*}$. Therefore, $g^{*}$ is a Cohen-Macaulay module over $B$ iff it is one over $A / I(g)$ and this is iff $g^{*}$ is a CohenMacaulay module over $A$. Now since $B$ is a regular algebra and $\operatorname{dim} B=\operatorname{dim}_{B} g^{*}$, the statement follows from the "freedom criterion" (GR] $)$ : a module over such an algebra is Cohen-Macaulay iff it is free.

1.3. Characteristic variety and complete intersections. A symbolic system $g$ is called an (algebraic) complete intersection if the algebra $S T / I(g)$ is such, i.e., if the ideal $I(g)$ is generated by $r=\operatorname{codim} \operatorname{Char}(g)$ elements (we mean codimension at the non-singular stratum).

A system $g$ is called a topological complete intersection if the variety $\operatorname{Char}(g)$ has this property, i.e., if it can be defined by the same number $r$ functions $f_{1}, \ldots, f_{r}$. Certainly the algebraic complete intersection condition implies the topological one, but the inverse is not true.

In the case of scalar equations $N \simeq \mathbb{R}$ the module $g^{*}=S T / I(g)$ is an algebra and the minimal number of generators for $I(g)$ is equal to the formal codimension $\operatorname{codim}(g)$ introduced in $\$ 1.1$. It is well-known that if the algebra $g^{*}$ is a complete intersection, then it is Cohen-Macaulay. In fact, one chooses a system $f_{1}, \ldots, f_{r} \in$ $I(g)$ defining $\operatorname{Char}(g)$. Since $r+\operatorname{dim} A /\left(f_{1}, \ldots, f_{r}\right) A=\operatorname{dim} A$, then (GR) the system can be extended to a system $f_{1}, \ldots, f_{n}$ of parameters of the algebra $A$. Then $f_{r+1}, \ldots, f_{n}$ is a maximal regular sequence.

Let's discuss the conditions of complete intersection in the general case $m=$ $\operatorname{dim} N \geq 1$. The symbolic system $g=\operatorname{Ker}\left(f: S T^{*} \otimes N \rightarrow \mathbb{R}^{r}\right)$ is given by $r=\operatorname{codim}(g)$ vector space $N^{*}$-valued polynomials $f_{1}, \ldots, f_{r}$. Let $e_{1}, \ldots, e_{m}$ be a basis in $N$. For every $p \in T^{*}$ we have the matrix $\Phi(p)=\left\|f_{i}^{j}(p)\right\|_{r \times m}$, where $f_{i}(p)=\sum_{j} f_{i}^{j}(p) e_{j}^{*}$. Then

$$
\operatorname{Char}(g)=\left\{p \in P^{\mathbb{C}} T^{*} \mid \operatorname{rank} \Phi(p)<\min (r, m)\right\} .
$$

For the determined systems $r=m$ the characteristic variety has codimension one. In the overdetermined case, we are interested in, $\operatorname{Char}(g)$ is defined by the equations $\operatorname{det}\left(\Delta_{\nu}\right)=0$ for all $m \times m$ minors in $\Phi$. This system of equations is, however, redundant: There are non-trivial linear relations among $\operatorname{det}\left(\Delta_{\nu}\right)$. 
Let's associate to every characteristic vector $p$ the space $\mathcal{K}_{p}=\operatorname{Ker} \Phi(p) \subset N$ (in the underdetermined case $r<m$, which we will not discuss here, one should consider the cokernel), which is the value of the module $g^{*}$ at $p \in T^{*}$. The union $\bigcup_{p} \mathcal{K}_{p}$ constitute the kernel sheaf over Char $(g)$. The base Char $(g)$ is stratified according to the fiber dimension and there is an open stratum on which the dimension of fibers is minimal. Denote this number by $k \in[\max (1, m-r), m]$.

Proposition 6. It is sufficient to have $l=k(r-m+k)$ equations defining Char $(g)$ locally near the points of open strata, in particular, codim $\operatorname{Char}(g) \leq l$.

Proof. We have $\operatorname{rank} \Phi(p) \leq m-k$. At a generic point a nondegenerate $(m-k) \times$ $(m-k)$ minor can be chosen. Now we should check that all $k(r-m+k)$ bordering $(m-k+1) \times(m-k+1)$ minors are degenerate.

Thus, for example, if $k=1$ (as for the scalar equations), $l=r-m+1$.

Corollary. Let the kernel sheaf have fibers of constant dimension $k$ and let some $(m-k) \times(m-k)$ minor in $\Phi$ be non-degenerate for all $p \in \operatorname{Char}(g)$. Then if codim $\operatorname{Char}(g)=k(r-m+k)$, the characteristic variety is a topological complete intersection.

Proof. Actually, there are $l=k(r-m+k)$ functions defining Char $(g)$.

The simplest necessary condition for $\operatorname{Char}(g)$ to be a complete intersection is local 1-connectedness ([Ha] $)$.

Remark 3. A system of PDEs can be represented in many equivalent forms. For example a scalar system can be always written as a system of several equations of the first order. The characteristic variety as well as the kernel sheaf over it are important invariants of the equation itself. For instance, if there are fibers of dimensions greater than 1 , the system is not reduced to a scalar one.

1.4. Differential equations. Let $\pi: E_{\pi} \rightarrow M$ be a smooth bundle over an $n$ dimensional manifold with fibers of dimension $m$ and $\pi_{k}: J^{k}(\pi) \rightarrow M$ the bundle consisting of $k$-jets of its sections. There are natural projections $\pi_{k, l}: J^{k}(\pi) \rightarrow$ $J^{l}(\pi)$. A PDEs system of pure order $k$ is usually represented as a smooth subbundle $\mathcal{E} \subset J^{k}(\pi)([\mathrm{KLV}])$. Scalar PDEs correspond to the trivial bundle $E_{\pi}=M \times \mathbb{R}$ and $\mathcal{E} \subset J^{k}(M)$.

To cover the case of several equations of different orders we modify the usual definition. By a differential equation/system of (maximal) order $k$ we mean a sequence $\mathcal{E}=\left\{\mathcal{E}_{l}\right\}_{-1 \leq l \leq k}$ of submanifolds $\mathcal{E}_{l} \subset J^{l}(\pi)$ with $\mathcal{E}_{-1}=M, \mathcal{E}_{0}=J^{0} M=$ $E_{\pi}$ such that for all $0<l \leq k$ the following conditions hold:

(1) $\pi_{l, l-1}^{\mathcal{E}}: \mathcal{E}_{l} \rightarrow \mathcal{E}_{l-1}$ are smooth fiber bundles.

(2) The first prolongations $\mathcal{E}_{l-1}^{(1)}$ are smooth subbundles of $\pi_{l}$ and $\mathcal{E}_{l} \subset \mathcal{E}_{l-1}^{(1)}$.

Consider a point $x_{k} \in \mathcal{E}_{k}$ with $x_{l}=\pi_{k, l}\left(x_{k}\right)$ for $l<k$ and $x=x_{-1}$. It determines a symbolic system $g \subset S T_{x}^{*} M \otimes N_{x}$, where $N_{x}=T_{x_{0}}\left[\pi^{-1}(x)\right]$, by the formula $g_{l}=T_{x_{l}}\left[\left(\pi_{l, l-1}^{\mathcal{E}}\right)^{-1}\left(x_{l-1}\right)\right] \subset S^{l} T_{x}^{*} M \otimes N_{x}$ for $l \leq k$ and $g_{l}=g_{k}^{(l-k)}$ for $l>k$. The conditions above imply that the symbols $g_{l}$ form smooth vector bundles over $\mathcal{E}_{l}$ and that $g_{l} \subset g_{l-1}^{(1)}$ for $l \leq k$.

The Spencer $\delta$-complex for PDEs system $\mathcal{E}$ at a point $x_{k} \in \mathcal{E}_{k}$ is the Spencer complex for its symbolic system at this point. The corresponding $\delta$-cohomology is denoted by $H^{i, j}\left(\mathcal{E} ; x_{k}\right)$ and we usually omit reference to the point. 
We define regular PDEs system of maximal order $k$ as a submanifold $\mathcal{E}=\mathcal{E}_{k} \subset$ $J^{k} M$ filtered by $\mathcal{E}_{l}$ and such that the symbolic system and the Spencer cohomology form graded bundles over it. We define orders $\operatorname{ord}(\mathcal{E})$ of the system and its formal codimension $\operatorname{codim}(\mathcal{E})$ as these quantities for the symbolic system.

Remark 4. A system of different PDEs of orders $k_{1}, \ldots, k_{r} \leq k$ has $\operatorname{ord}(\mathcal{E})=$ $\left\{k_{1}, \ldots, k_{r}\right\}$ (multiple orders repeat) if there are no relations of order $k$ between the equations. The number $r=\operatorname{codim}(\mathcal{E})$ of involved PDEs is an important invariant of the system. If $r>m$, the system is called overdetermined, in the case of equality determined and otherwise $\mathcal{E}$ is underdetermined.

In order to describe the first obstruction to prolong $\mathcal{E}$ we briefly recall some fundamental geometric notions of the jet-spaces (see KLV, Ly, KL1] for details). Denote by $L\left(x_{k}\right) \subset T_{x_{k-1}} J^{k-1}(\pi)$ the tangent space $T_{x_{k-1}}\left[j_{k-1}(s)(M)\right]$ to the jetextension of a section $s \in C_{\mathrm{loc}}^{\infty}(\pi)$ with $j_{k}(s)(x)=x_{k}$ (this subspace does not depend on a particular choice of $s$, but only on $x_{k}$ ).

Define the Cartan distribution on the space $J^{k}(\pi)$ by the formula $\mathcal{C}_{k}\left(x_{k}\right)=$ $\left(d \pi_{k, k-1}\right)^{-1} L\left(x_{k}\right)$. It induces the Cartan distribution on $\mathcal{E}_{k}: \mathcal{C}_{\mathcal{E}_{k}}=\mathcal{C}_{k} \cap T \mathcal{E}_{k}$. Cartan connection on $\mathcal{E}_{k}$ is a horizontal subdistribution in it, i.e., a smooth family $H\left(x_{k}\right) \subset \mathcal{C}_{\mathcal{E}_{k}}\left(x_{k}\right), x_{k} \in \mathcal{E}_{k}$, such that $d \pi_{k}: H\left(x_{k}\right) \rightarrow T_{x} M$ is an isomorphism. Having a Cartan connection we can decompose $\mathcal{C}_{\mathcal{E}_{k}}\left(x_{k}\right) \simeq H\left(x_{k}\right) \oplus g_{k}\left(x_{k}\right)$.

Given a distribution $\Pi$ on a manifold its derived differential system $\partial \Pi$ is generated by the commutators of its sections. In the regular case it is a distribution and one gets the effective normal bundle $\nu=\partial \Pi / \Pi$. The curvature of $\Pi$ is the vector-valued 2-form $\Xi_{\Pi} \in \Lambda^{2} \Pi^{*} \otimes \nu$ given by the formula

$$
\Xi_{\Pi}(\xi, \eta)=[\xi, \eta] \bmod \Pi, \quad \xi, \eta \in \Pi
$$

(although the formula uses vector fields, the tensor $\Xi_{\Pi}$ depends only on their values at the considered point).

The metasymplectic structure $\Omega_{k}$ on $J^{k}(\pi)$ is the curvature of the Cartan distribution. At a point $x_{k}$ it is a 2 -form on $\mathcal{C}_{k}\left(x_{k}\right)$ with values in the vector space $F_{k-1}\left(x_{k-1}\right)=T_{x_{k-1}}\left[\pi_{k-1, k-2}^{-1}\left(x_{k-2}\right)\right] \simeq S^{k-1} T_{x}^{*} M \otimes N_{x}$. To describe it fix a point $x_{k+1} \in J^{k+1}(\pi)$ over $x_{k}$ and decompose $\mathcal{C}_{k}\left(x_{k}\right)=L\left(x_{k+1}\right) \oplus F_{k}\left(x_{k}\right)$. Then $\Omega_{k}(\xi, \eta)=0$ if both $\xi, \eta$ belong simultaneously either to $L\left(x_{k+1}\right)$ or to $F_{k}\left(x_{k}\right)$. But if $\xi \in L\left(x_{k+1}\right)$ corresponds to $X=d \pi_{k}(\xi) \in T_{x} M$ and $\eta \in F_{k}\left(x_{k}\right)$ corresponds to $\theta \in S^{k} T_{x}^{*} M \otimes N_{x}$, then the value of $\Omega_{k}(\xi, \eta)$ equals

$$
\Omega_{k}(X, \theta)=\delta_{X} \theta \in S^{k-1} T_{x}^{*} M \otimes N_{x},
$$

where $\delta_{X}=i_{X} \circ \delta$ is the differentiation along $X$. The introduced structure does not depend on the point $x_{k+1}$ determining the decomposition because the subspace $L\left(x_{k+1}\right)$ is $\Omega_{k}$-isotropic.

Restriction of the metasymplectic structure $\Omega_{k} \in F_{k-1} \otimes \Lambda^{2} \mathcal{C}_{k}^{*}$ to the equation is the tensor $\Omega_{\mathcal{E}_{k}} \in g_{k-1} \otimes \Lambda^{2} \mathcal{C}_{\mathcal{E}_{k}}^{*}$. Given a Cartan connection $H$ we define its curvature at $x_{k}$ to be $\left.\Omega_{\mathcal{E}_{k}}\right|_{H\left(x_{k}\right)} \in g_{k-1} \otimes \Lambda^{2} T_{x}^{*} M$. Considered as an element of the Spencer complex, it is $\delta$-closed and a change of the Cartan connection effects in a shift by a $\delta$-exact element.

The Weyl tensor $W_{k}\left(\mathcal{E} ; x_{k}\right)$ of the PDEs system $\mathcal{E}$ is the $\delta$-cohomology class $\left[\left.\Omega_{\mathcal{E}_{k}}\right|_{H\left(x_{k}\right)}\right] \in H^{k-1,2}\left(\mathcal{E} ; x_{k}\right)$. For geometric structures represented as PDEs systems it coincides with the classical structural function. 
Define the prolongation of the equation $\mathcal{E}_{k}$ by the formula

$$
\mathcal{E}_{k+1}:=\mathcal{E}_{k}^{(1)}=\left\{x_{k+1} \in J^{k+1}(\pi) \mid L\left(x_{k+1}\right) \subset \mathcal{C}_{\mathcal{E}_{k}}\left(x_{k}\right)\right\} .
$$

Let us call the prolongation regular if $\pi_{k+1, k}^{\mathcal{E}}: \mathcal{E}_{k+1} \rightarrow \mathcal{E}_{k}$ is a bundle map. For regular systems a necessary and sufficient condition for regularity of the first prolongation is vanishing of the Weyl tensor $W_{k}(\mathcal{E})=0$.

A system of different orders should be investigated for formal integrability successively by the maximal order $k$. If some prolongation $\mathcal{E}_{k}^{(1)}$ is not regular, its projections $\left\{\pi_{k+1, l}\left(\mathcal{E}_{k+1}\right)\right\}_{l \leq k}$, form a new system of maximal order $k$. Taking the regular part one continues with prolongations. The process stops in a finite number of steps. In fact, the statement about finite formal codimension of $g$ from $\$ 1.1$ becomes in the PDEs context the celebrated Cartan-Kuranishi theorem on prolongations: There exists a number $k_{0}$ such that $\mathcal{E}_{k}^{(1)}=\mathcal{E}_{k+1}$ for all $k \geq k_{0}$.

Definition 3. Call a regular PDEs system $\mathcal{E} \subset J^{k} M$ a complete intersection or Cohen-Macaulay respectively if the corresponding symbolic system $g$ is such.

A scalar system $\mathcal{E}$ is a complete intersection if it can be represented by differential equations $F_{1}=0, \ldots, F_{r}=0$ and the characteristic varieties, given by the symbols $f_{i}=0,1 \leq i \leq r$, are transversal. In particular, $\operatorname{Char}_{x_{k}} \mathcal{E} \subset P^{\mathbb{C}} T_{x}^{*} M$ has codimension $r=\operatorname{codim}(\mathcal{E})$.

Example of non-complete intersection is provided by the following system of formal codimension 2: $\left\{u_{x x}=0, u_{x y}=0\right\}$. Note that it is equivalent to the 1parametric $\operatorname{PDE}\left\{u_{x}=c\right\}$ of codim $=1$.

1.5. Examples. A PDEs system $\mathcal{E}$ with constant coefficients on a vector space $T$ has the same symbols at all of its points $x_{k} \in \mathcal{E}_{k}$. We will identify $T$ and its tangent spaces $T_{x}(T)$. Thus we identify a symbolic system $g$ with some constant coefficient system of differential equations.

In this subsection we demonstrate certain counterexamples to extensions of Theorem A, which show the assumption of the theorem is essential.

1. Consider the following first order system of partial differential equations

$$
\mathcal{E}=\left\{\frac{\partial u}{\partial x}=0, \frac{\partial v}{\partial x}=0, \frac{\partial u}{\partial z}=\frac{\partial v}{\partial y}\right\}
$$

on the space $T=V \oplus W$, where $V=\mathbb{R}^{1}(x), W=\mathbb{R}^{2}(y, z)$. Let $g$ be the corresponding symbolic system $g_{i+1}=g_{1}^{(i)}$. The module $g^{*}$ is isomorphic to $S^{1+\bullet} W:=W \cdot S W$, with the module structure induced from the projection along $V$ homomorphism $S T \rightarrow S W$. We have

$$
I(g)=S V \subset S T \text { and } \operatorname{Char}(g)=P^{\mathbb{C}} W^{*}=\left\{p_{x}=0\right\} \simeq \mathbb{C} P^{1} \subset \mathbb{C} P^{2} .
$$

So $g$ is a complete intersection. However, since $\operatorname{dim} g^{*}=\operatorname{dim} W=2$ and $S^{1+\bullet} W=$ $\bigoplus_{i>0} S^{i} W$ is not a free $S W$-module, it is not Cohen-Macaulay (see Proposition [5). Thus $\mathcal{E}$ is not Cohen-Macaulay.

The $V^{*}$-reduction $\tilde{g}_{i}=g_{i} \cap S^{i} V^{*}=0$ for $i>0$ and so the Euler characteristic for the order 2 Spencer $\delta$-complex equals $\tilde{\chi}=0$. On the other hand, the complex

$$
0 \rightarrow g_{2} \rightarrow g_{1} \otimes T^{*} \rightarrow \mathbb{R}^{2}(u, v) \otimes \Lambda^{2} T^{*} \rightarrow 0
$$

has $\chi=1$. Thus the Spencer cohomologies of $g$ and $\tilde{g}$ are different and we see the importance of the condition in Theorem $\mathrm{A}$. 
2. Consider a symmetric matrix $A=\left[\begin{array}{ll}u_{11} & u_{12} \\ u_{12} & u_{22}\end{array}\right]$ depending on a point of $W=$ $\mathbb{R}^{2}(y, z)$. Equation $\operatorname{Hess}(u)=A$ has the compatibility condition

$$
\mathcal{E}=\left\{\frac{\partial u_{11}}{\partial z}=\frac{\partial u_{12}}{\partial y}, \frac{\partial u_{12}}{\partial z}=\frac{\partial u_{22}}{\partial y}\right\} .
$$

We extend this system constantly along $x$ to $T=V \oplus W$ as above and note that the corresponding symbolic system has non-free dual $g^{*} \simeq S^{2+} \bullet W$ and hence cannot be Cohen-Macaulay. Therefore, we obtain other counterexamples to generalizations of Theorem A.

3. Consider the following system of scalar PDEs on $T=\mathbb{R}^{4}(x, y, z, t)$ :

$$
\mathcal{E}=\left\{\frac{\partial^{2} u}{\partial x \partial z}=\frac{\partial^{2} u}{\partial y^{2}}, \frac{\partial^{2} u}{\partial y \partial t}=\frac{\partial^{2} u}{\partial z^{2}}, \frac{\partial^{2} u}{\partial x \partial t}=\frac{\partial^{2} u}{\partial y \partial z}\right\}
$$

Its characteristic set is the well-known Veronese variety

$$
\operatorname{Char}(g)=\left\{\left[z_{0}: z_{1}: z_{2}: z_{3}\right] \in \mathbb{C} P^{3} \mid z_{0} z_{2}=z_{1}^{2}, z_{1} z_{3}=z_{2}^{2}, z_{0} z_{3}=z_{1} z_{2}\right\} .
$$

It is irreducible, has codimension 2 and its ideal $I(g)$ is not a complete intersection. Note though that the variety itself is a topological complete intersection since it is given by two equations: $z_{1}^{2}=z_{0} z_{2}$ and $z_{2}^{3}+z_{0} z_{3}^{2}=2 z_{1} z_{2} z_{3}$.

Calculations show that for the third order Spencer complex

$$
0 \rightarrow g_{3} \rightarrow g_{2} \otimes T^{*} \rightarrow g_{1} \otimes \Lambda^{2} T^{*} \rightarrow \Lambda^{3} T^{*} \rightarrow 0,
$$

$\operatorname{dim} g_{1}=4, \operatorname{dim} g_{2}=7, \operatorname{dim} g_{3}=10$. So the Euler characteristic equals $\chi=2$.

Consider the two choices of $V^{*}: V^{\prime *}=\left\{z_{3}=0\right\}$ and $V^{\prime \prime *}=\left\{z_{0}=0, z_{3}=0\right\}$. Both subspaces are transversal and we have $\operatorname{Char}(g) \cap P^{\mathbb{C}} V^{*}=\{[1: 0: 0: 0]\}$ or $\emptyset$ respectively. One calculates $\operatorname{dim} \tilde{g}_{1}^{\prime}=3, \operatorname{dim} \tilde{g}_{2}^{\prime}=5, \operatorname{dim} \tilde{g}_{3}^{\prime}=7$ and $\operatorname{dim} \tilde{g}_{1}^{\prime \prime}=2$, $\operatorname{dim} \tilde{g}_{2}^{\prime \prime}=3, \operatorname{dim} \tilde{g}_{3}^{\prime \prime}=4$. Consequently, the Euler characteristics of the reductions are $\tilde{\chi}^{\prime}=\tilde{\chi}^{\prime \prime}=0$.

Thus the system $g$ is a topological but not an algebraic complete intersection and the corollary of Theorem $\mathrm{A}$ is not valid for this case.

\section{Spencer cohomologies of Cohen-Macaulay SYMBOLIC SYSTEMS}

2.1. Reductions and filtration of symbols. Consider a vector space $T^{n}$ with a subspace $W^{n-m}$ and the quotient space $V^{m}=T^{n} / W^{n-m}$. We have the following exact sequence for the duals:

$$
0 \rightarrow\left[V^{*} \simeq \operatorname{ann}(W)\right] \hookrightarrow T^{*} \rightarrow W^{*} \rightarrow 0
$$

Consider a symbolic system $\left\{g_{k}\right\}$ on $T$ with values in a vector space $N$. Let us define $\tilde{g}_{k}=g_{k} \cap\left(S^{k} V^{*} \otimes N\right)$ and $g_{k}^{W}=\operatorname{Im}\left(g_{k} \rightarrow S^{k} W^{*} \otimes N\right)$.

Lemma 1. Sequences of subspaces $\tilde{g}$ and $g^{W}$ are symbolic systems.

Proof. We have $\delta: \tilde{g}_{k} \rightarrow\left(g_{k-1} \otimes T^{*}\right) \cap\left(S^{k-1} V^{*} \otimes N \otimes V^{*}\right)=\tilde{g}_{k-1} \otimes V^{*}$. Similarly, $\delta: g_{k}^{W} \rightarrow \operatorname{Im}\left(g_{k} \otimes T^{*} \rightarrow S^{k-1} W^{*} \otimes N \otimes W^{*}\right)=g_{k-1}^{W} \otimes W^{*}$.

Definition 4. The symbolic system $\tilde{g}$ is called the $V^{*}$-reduction. 
Let us define the filtration in $S^{l} T^{*}$ according to the symmetric powers of $V^{*}$. It induces the following decreasing filtrations in the symbols

$$
0 \subset \mathfrak{F}_{l}^{l} \subset \mathfrak{F}_{l-1}^{l} \subset \cdots \subset \mathfrak{F}_{0}^{l}=g_{l}, \quad \mathfrak{F}_{i}^{l}=g_{l} \cap\left(S^{i} V^{*} \cdot S^{l-i} T^{*} \otimes N\right) .
$$

We extend the filtration to arbitrary $i \in \mathbb{Z}$ by letting $\mathfrak{F}_{i}^{l}=\mathfrak{F}_{0}^{l}$ for $i<0$ and $\mathfrak{F}_{i}^{l}=0$ for $i>l$. Let us denote the associated graded group by

$$
\mathfrak{f}^{l}=\bigoplus \mathfrak{f}_{i}^{l} ; \quad \mathfrak{f}_{i}^{l}=\mathfrak{F}_{i}^{l} / \mathfrak{F}_{i+1}^{l} .
$$

Note that

$$
\mathfrak{f}_{i}^{l} \subset \frac{S^{i} V^{*} \cdot S^{l-i} T^{*} \otimes N}{S^{i+1} V^{*} \cdot S^{l-i-1} T^{*} \otimes N} \simeq S^{i} V^{*} \otimes S^{l-i} W^{*} \otimes N .
$$

There is an action $\delta_{w}: \mathfrak{F}_{i}^{l} \rightarrow \mathfrak{F}_{i}^{i}=\tilde{g}_{i}$ for every element $w \in S^{l-i} W$ (we have already used it in 1.2 . Since $\mathfrak{F}_{i+1}^{l}$ belongs to the kernel, one gets an injective map

$$
\varkappa_{i}^{l}: \mathfrak{f}_{i}^{l} \hookrightarrow \tilde{g}_{i} \otimes S^{l-i} W^{*} .
$$

Denote by $\bar{\delta}=\delta^{W}$ the Spencer $\delta$-differential acting by $W$.

Proposition 7. We have the following diagram of commuting $W$-complexes:

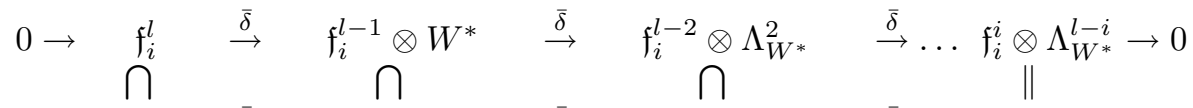

$$
\begin{aligned}
& 0 \rightarrow \tilde{g}_{i} \otimes S_{W^{*}}^{l-i} \stackrel{\bar{\delta}}{\rightarrow} \tilde{g}_{i} \otimes S_{W^{*}}^{l-i-1} \otimes W^{*} \stackrel{\bar{\delta}}{\rightarrow} \tilde{g}_{i} \otimes S_{W^{*}}^{l-i-2} \otimes \Lambda_{W^{*}}^{2} \stackrel{\bar{\delta}}{\rightarrow} \ldots \tilde{g}_{i} \otimes \Lambda_{W^{*}}^{l-i} \rightarrow 0,
\end{aligned}
$$

where the vertical inclusions are induced by the maps $\varkappa_{i}^{l}$ and we introduce the notation $S_{W^{*}}^{t}=S^{t} W^{*}, \Lambda_{W^{*}}^{t}=\Lambda^{t} W^{*}$ for compactness of the diagram.

Proof. From the commutative diagram

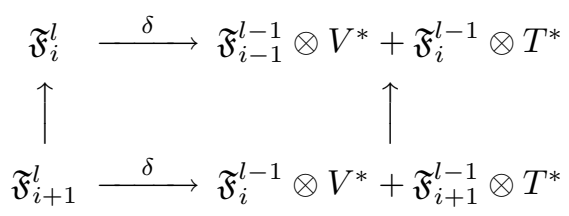

we obtain the following description of the differential in the upper complex:

$$
\mathfrak{f}_{i}^{l} \stackrel{\delta}{\longrightarrow} \frac{\mathfrak{F}_{i-1}^{l-1} \otimes V^{*}+\mathfrak{F}_{i}^{l-1} \otimes T^{*}}{\mathfrak{F}_{i}^{l-1} \otimes V^{*}+\mathfrak{F}_{i+1}^{l-1} \otimes T^{*}}=\left[\frac{\mathfrak{F}_{i-1}^{l-1}}{\mathfrak{F}_{i}^{l-1}} \otimes V^{*}\right] \oplus\left[\frac{\mathfrak{F}_{i}^{l-1}}{\mathfrak{F}_{i+1}^{l-1}} \otimes W^{*}\right] \stackrel{\text { proj }}{\longrightarrow} \mathfrak{f}_{i}^{l-1} \otimes W^{*},
$$

i.e., $\bar{\delta}=\operatorname{proj} \circ \delta$. Now the commutativity of the diagram is easily checked on the generators. In fact, if $\sum p_{i_{1} \ldots i_{t}} w_{i_{1}} \wedge \ldots \wedge w_{i_{t}}$ is an element of $\mathfrak{F}_{i}^{s} \otimes \Lambda^{t} W^{*}$, then both $\varkappa \bar{\delta}$ and $\bar{\delta} \varkappa$ map it into

$$
\sum \delta_{w_{j_{0}} w_{j_{1}} \ldots w_{j_{s-i-1}}}\left(p_{i_{1} \ldots i_{t}}\right) \cdot w_{j_{1}} \cdots w_{j_{s-i-1}} \otimes w_{j_{0}} \wedge w_{i_{1}} \wedge \ldots \wedge w_{i_{t}} .
$$

In the next section we show how the cohomology groups of the Spencer $W$ complex $\left(\mathfrak{f}_{i}, \bar{\delta}\right)$ occur. 
2.2. Spectral sequence for the Spencer cohomology. Consider the following filtration in $\Lambda^{s} T^{*}$ :

$$
0 \subset \lambda_{s}^{s} \subset \lambda_{s-1}^{s} \subset \cdots \subset \lambda_{0}^{s}=\Lambda^{s} T^{*}, \quad \lambda_{j}^{s}=\Lambda^{j} V^{*} \wedge \Lambda^{s-j} T^{*} .
$$

It produces a filtration in the $l$-th Spencer complex:

$$
F^{p, q}=g_{l-p-q} \otimes \lambda_{p}^{p+q} .
$$

Lemma 2. The filtration is monotone decreasing, $F^{p+1, q-1} \subset F^{p, q}$, and is preserved by the $\delta$-map, $\delta F^{p, q} \subset F^{p, q+1}$.

This filtration determines the spectral sequence of Leray-Serre type with

$$
E_{0}^{p, q}=F^{p, q} / F^{p+1, q-1}=g_{l-p-q} \otimes \Lambda^{p} V^{*} \otimes \Lambda^{q} W^{*} .
$$

The differential $d_{0}: E_{0}^{p, q} \rightarrow E_{0}^{p, q+1}$ acts by $W$ and so

$$
E_{1}^{p, q}=H^{l-p-q, q}(g, \bar{\delta}) \otimes \Lambda^{p} V^{*}
$$

To calculate the $\bar{\delta}$-Spencer cohomology of $g_{l}$ we introduce another spectral sequence (all terms marked with bar). To construct it we use the filtration of the previous section and define $\bar{F}^{a, b}=\mathfrak{F}_{a}^{l-a-b} \otimes \Lambda^{a+b} W^{*}$. This filtration is again decreasing and $\bar{\delta}$-preserved. The 0 -term of the new spectral sequence is

$$
\bar{E}_{0}^{a, b}=\bar{F}^{a, b} / \bar{F}^{a+1, b-1}=\left[\mathfrak{F}_{a}^{l-a-b} / \mathfrak{F}_{a+1}^{l-a-b}\right] \otimes \Lambda^{a+b} W^{*}=\mathfrak{f}_{a}^{l-a-b} \otimes \Lambda^{a+b} W^{*}
$$

and so $\bar{E}_{1}^{a, b}=H^{l-a-b, a+b}\left(\mathfrak{f}_{a}, \bar{\delta}\right)$. Let us show now how this spectral sequence can be calculated in two special cases.

1. Involutive case. Let $k_{a}+1$ be the minimal number $k \geq 0$ such that $\mathfrak{f}_{a}^{a+k} \neq \tilde{g}_{a} \otimes S^{k} W^{*}$, certainly $k_{a} \geq 0$ for every $a$ (the case $k_{a}=+\infty$ corresponds to the $\bar{\delta}$-acyclic complex). The system $\mathfrak{f}_{a}$ is called $\bar{\delta}$-involutive if the only Spencer cohomology occurs at the term $\tilde{g}_{a} \otimes S^{k_{a}} W^{*} \otimes \Lambda^{l-a-k_{a}} W^{*}$ (note that this is involutivity along $W$ only). Denote this cohomology by $h_{a}^{l}=H^{a+k_{a}, l-a-k_{a}}\left(\mathfrak{f}_{a}, \bar{\delta}\right)$. Thus we have

$$
\bar{E}_{1}^{a, b}= \begin{cases}h_{a}^{l}, & b=l-2 a-k_{a} \\ 0, & \text { else. }\end{cases}
$$

Suppose now $k_{a} \equiv k$ for all $a$. Then the differentials $\bar{d}_{r}: \bar{E}_{r}^{a, b} \rightarrow \bar{E}_{r}^{a+r, b-r+1}$ are trivial by dimensional reasons and so $\bar{E}_{\infty}^{a, b}=\bar{E}_{1}^{a, b}$ and

$$
H^{l-j, j}(g, \bar{\delta}) \simeq \bigoplus_{a+b=j} \bar{E}_{\infty}^{a, b}=\bigoplus_{a+(l-2 a-k)=j} h_{a}^{l}=h_{l-j-k}^{l} .
$$

Therefore $E_{1}^{p, q}=h_{l-p-q-k}^{l-p} \otimes \Lambda^{p} V^{*}$. Note that in many cases when the most groups $h_{t}^{s}$ vanish or $\operatorname{dim} V$ is small, the differentials $d_{r}, r>0$, and the term $E_{\infty}^{p, q}$ can be calculated.

2. Split case. Suppose $\mathfrak{f}_{a}^{l} \simeq \tilde{g}_{a} \otimes g_{l-a}^{W}$. Then

$$
\bar{E}_{1}^{a, b}=\tilde{g}_{a} \otimes H^{l-2 a-b, a+b}\left(g^{W}, \delta^{W}\right) .
$$

The differentials $\bar{d}_{r}$ are trivial due to their definitions and so $\bar{E}_{\infty}^{a, b}=\bar{E}_{1}^{a, b}$. Thus

$$
H^{l-j, j}(g, \bar{\delta}) \simeq \bigoplus_{a+b=j} \bar{E}_{\infty}^{a, b}=\bigoplus_{a} \tilde{g}_{a} \otimes H^{l-a-j, j}\left(g^{W}, \delta^{W}\right) .
$$


So $E_{1}^{p, q}=\bigoplus_{a+b=l-p-q}\left(\tilde{g}_{a} \otimes \Lambda^{p} V^{*}\right) \otimes H^{b, q}\left(g^{W}, \delta^{W}\right)$ and we compute

$$
E_{2}^{p, q}=\bigoplus_{a+b=l-p-q} H^{a, p}\left(\tilde{g}, \delta^{V}\right) \otimes H^{b, q}\left(g^{W}, \delta^{W}\right) .
$$

Since the $E_{2}$ term is split the sequence stabilizes $E_{\infty}^{p, q}=E_{2}^{p, q}$ and we find the Spencer cohomology

$$
H^{l-j, j}(g, \delta)=\bigoplus_{p+q=j} E_{\infty}^{p, q}=\bigoplus_{a+b=l-j} \bigoplus_{p+q=j} H^{a, p}\left(\tilde{g}, \delta^{V}\right) \otimes H^{b, q}\left(g^{W}, \delta^{W}\right) .
$$

Remark 5. The first considered case is analogous to the Guillemin's involutivity theorem $\mathrm{Gu}$ and leads to a similar statement. The second occasion arises in the reduced systems if we choose $W$ of codimension less than $\operatorname{dim}(g)$. Then the projection of Char $(g)$ along $V^{*}$ is not surjective and we get a symbolic system $g^{W}$. In particular, this case generalizes our Theorem $\mathrm{A}$.

2.3. Proof of Theorem A. A subspace $V^{*} \subset T^{*}$ is called non-characteristic if its (complexified) projectivization does not intersect the characteristic variety. Let $\operatorname{codim} \operatorname{Char}(g)=r$. The following statement holds for general symbolic systems.

Proposition 8. The set of non-characteristic subspaces $V^{*} \subset T^{*}$ of dimension $s \leq r$ is open and dense in $\operatorname{Gr}_{s}\left(T^{*}\right)$. For each such a subspace the $V^{*}$-reduced system $\tilde{g}$ is of finite type $\tilde{g}_{k}=0$ for $k>k_{0}$.

Proof. It is enough to prove the statement for $s=r$. By Noether normalization lemma (Mum) for every subvariety $X$ in $\mathbb{C} P^{n-1}$ of codimension $r$ there is a (complex) linear subspace $L$ of dimension $r-1$ such that $L \cap X=\emptyset$ and, moreover, a.e. subspace $L$ is such. Take $X=\operatorname{Char}(g) \subset P^{\mathbb{C}} T^{*}$. The above $L$ determines a real subspace $V^{*} \subset T^{*}$ of dimension equal to $r$. Reduction $\tilde{g}$ has no characteristics and hence is of finite type.

Remark 6. For Cohen-Macaulay systems the above number $k_{0}$ is important, since starting from it $g$ becomes involutive (thanks to the referee for this remark). In general, it can be estimated roughly from the Poincaré $\delta$-lemma ( $\underline{\mathrm{S}}, \underline{\mathrm{KLV}}]$ ) for $\tilde{g}$ and even this gives a better estimate than the standard estimate for the system $g$ (on higher-dimensional space: $\operatorname{dim} T>\operatorname{dim} V$ ). But in certain cases, one can find this number explicitly. For example, if $g$ is a complete intersection system defined by differential equations of orders $r_{1}, \ldots, r_{s}$, then $k_{0}=r_{1}+\cdots+r_{s}-1$. Thus Theorem A facilitates investigation of formal integrability of an overdetermined Cohen-Macaulay system by reducing it to a finite type system.

Proof of Theorem $\mathrm{A}$. We consider at first the situation, when $V^{*}$ is non-characteristic of dimension $r$. We use Theorem 1.7 of $\mathrm{AB}$. It states that if an a-module $\mathrm{E}$ and two ideals $\mathfrak{c} \subset \mathfrak{b} \subset \mathfrak{a}$ satisfy $\mathfrak{c}+\operatorname{ann}(\mathrm{E}) \supset \mathfrak{b}^{k}$ for some $k>0$, then all the Koszul homologies $H_{q}(\overline{\mathrm{E}})$ vanish for $q>q_{0}=\operatorname{dim} \mathrm{E}-\operatorname{depth} \mathrm{E}$ and the $q_{0}$-homology is nonzero. Here $\bar{E}$ the space $E$ considered as a $\mathfrak{c}$-module.

In our case $\mathrm{E}=g^{*}, \mathfrak{a}=S T, \mathfrak{b}=S^{1+\bullet} T, \mathfrak{c}=W \cdot S T$. The condition on $\mathfrak{b}^{k}$ follows from our choice of $V^{*}$ and the Noether theorem. The Cohen-Macaulay assumption for $g^{*}$ implies $q_{0}=0$. Note that the same statement follows from the fact that $g^{*}$ is a free $S W$-module.

Now we use the duality between Koszul and Spencer complexes and obtain that the only nonzero cohomology group of the $W$-complex is $H^{l, 0}(g, \bar{\delta})=\tilde{g}_{l}$. The 1st 
term of the spectral sequence for the Spencer cohomology has support in the line $q=0$, on which $E_{1}^{p, 0}=\tilde{g}_{l-p} \otimes \Lambda^{p} V^{*}$. Thus $E_{2}^{p, 0}=H^{l-p, p}(\tilde{g})$ is the only nonzero cohomology of the second term and the sequence stabilizes, whence

$$
H^{l-j, j}(g)=\bigoplus_{p+q=j} E_{\infty}^{p, q}=E_{2}^{j, 0}=H^{l-j, j}(\tilde{g}) .
$$

Finally, if $V^{*}$ is transversal of dimension $>r$, then there exists a non-characteristic subspace $V_{0}^{*} \subset V^{*}$. Actually, we apply the Noether normalization to the codimension $r$ variety $\operatorname{Char}(g) \cap P^{\mathbb{C}} V^{*}$ in $P^{\mathbb{C}} V^{*}$. Now by the argumentation of $₫ 1.2$ (Proposition 5) the symbolic system $\tilde{g}=g \cap S V^{*} \otimes N$ is Cohen-Macaulay provided $g$ is. Let us denote $\tilde{g}^{0}$ the $V_{0}^{*}$-reduction of $g$ (and also of $\tilde{g}$ ). Then by the above, the Spencer $\delta$-cohomologies of $g$ and $\tilde{g}^{0}$ coincide; but for the same reason the Spencer $\delta$-cohomologies of $\tilde{g}$ and $\tilde{g}^{0}$ coincide also. The theorem is proved.

In the above proof we used a theorem of Auslander and Buchsbaum. Their paper $\mathrm{AB}$ ] concerns mainly local algebras. The statement in the formal case can be found in Serre's letter reproduced in [GS]. We use it for $q_{0}>0$ to get:

Proposition 9. The term $E_{1}^{p, q}$ of the spectral sequence constructed in 92.2 has support in the first $\left(q_{0}+1\right)$-rows $0 \leq q \leq q_{0}$, where $q_{0}=\operatorname{dim} g^{*}-\operatorname{depth} g^{*}$. Thus the sequence stabilizes at the term $E_{2+q_{0}}$.

Remark 7. For a transversal $V^{*}$ the subspace $g_{k}$ is transversal to $g_{k-1}^{(1)} \cap S^{k} V^{*}$ in $g_{k-1}^{(1)}$. This is equivalent to the surjectivity of the map $\delta_{w}=i_{w} \circ \delta: g_{k} \rightarrow \delta\left(g_{k-1}^{(1)}\right) \subset$ $g_{k-1}$ for all nonzero $w \in W$. Actually in 22.4 we will prove more, namely the surjectivity of $\delta_{w}: g_{k} \rightarrow g_{k-1}$ for $w \in W \backslash\{0\}$.

It is interesting to compare this with a criteria of involutivity (GS]) for the first order PDEs systems: $g_{1} \subset T^{*} \otimes N$ is involutive iff there exists a filtration $\{0\}=$ $W_{0} \subset \ldots W_{i} \subset \ldots W_{n}=T$ with $\operatorname{dim} W_{i}=i$ such that for $g[i]=g_{1} \cap \operatorname{ann}\left(W_{i}\right) \otimes N$ the map $\delta_{w}: g[i]^{(1)} \rightarrow g[i]$ is epimorphic for some $w \in W_{i+1} \backslash W_{i}$.

2.4. Dualization. In this section we give another idea of calculation of the $\bar{E}$ spectral sequence. We restrict for simplicity to the case of scalar systems $(N=\mathbb{R})$, which are complete intersections, and to the non-characteristic $V^{*}$ of maximal dimension $r=\operatorname{codim} \operatorname{Char}(g)$. We will prove that the maps $\varkappa_{i}^{l}$ in (2) are epimorphisms and thus isomorphisms.

Lemma 3. For a transversal $V^{*}$ we have $\mathfrak{F}_{0}^{l} / \mathfrak{F}_{1}^{l} \stackrel{\varkappa}{\simeq} S^{l} W^{*}$.

Proof. Due to our choice of $V^{*}$ and the Noether normalization lemma we have an epimorphism $\operatorname{proj}_{V^{*}}: \operatorname{Char}(g) \rightarrow P^{\mathbb{C}} W^{*}$. So for every $\theta \in W^{*}$ there exists $\zeta \in \operatorname{Char}(g)$ with $\operatorname{proj}_{V^{*}}(\zeta)=\theta$. Therefore the restriction $\operatorname{proj}_{V^{*}}^{l}: g_{l} \rightarrow S^{l} W^{*}$ is epimorphic. Since the kernel is $g_{l} \cap V^{*} \cdot S^{l-1} T^{*}=\mathfrak{F}_{1}^{l}$, the claim follows from the exact sequence

$$
0 \rightarrow \mathfrak{F}_{1}^{l} \rightarrow \mathfrak{F}_{0}^{l} \rightarrow S^{l} W^{*} \rightarrow 0
$$

To cover the case $i>0$ we act as follows. Taking the annulator of

$$
S^{l} T^{*}=\mathfrak{F}_{-1}^{l} \supset \mathfrak{F}_{0}^{l} \supset \mathfrak{F}_{1}^{l} \cdots \supset \mathfrak{F}_{l}^{l} \supset \mathfrak{F}_{l+1}^{l}=0 .
$$

we get the filtration

$$
0=\mathfrak{H}_{-1}^{l} \subset \mathfrak{H}_{0}^{l} \subset \mathfrak{H}_{1}^{l} \subset \ldots \mathfrak{H}_{l}^{l} \subset \mathfrak{H}_{l+1}^{l}=S^{l} T,
$$


where

$$
\mathfrak{H}_{i}^{l}=\operatorname{ann}\left(g_{l} \cap S^{i} V^{*} \cdot S^{l-i} T^{*}\right)=\operatorname{ann}\left(g_{l}\right)+\operatorname{ann}\left(S^{i} V^{*} \cdot S^{l-i} T^{*}\right)=I_{l}^{(0)}+\mathfrak{G}_{i}^{l}
$$

and $\mathfrak{G}_{i}^{l}=S^{l-i+1} W \cdot S^{i-1} T$ (for $I_{l}^{(0)}$ see $\$ 1.2$ ). Due to the commutative diagram

$$
\begin{array}{llll}
\mathfrak{H}_{i}^{l} & \subset & \mathfrak{H}_{i+1}^{l} \\
\cup & & \cup \\
\mathfrak{G}_{i}^{l} & \subset & \mathfrak{G}_{i+1}^{l}
\end{array}
$$

we have a well-defined map

$$
S^{i} V \otimes S^{l-i} W \simeq \mathfrak{G}_{i+1}^{l} / \mathfrak{G}_{i}^{l} \stackrel{\phi}{\longrightarrow} \mathfrak{H}_{i+1}^{l} / \mathfrak{H}_{i}^{l}=\frac{\operatorname{ann}\left(\mathfrak{F}_{i+1}^{l}\right)}{\operatorname{ann}\left(\mathfrak{F}_{i}^{l}\right)} \simeq\left(\mathfrak{F}_{i}^{l} / \mathfrak{F}_{i+1}^{l}\right)^{*}
$$

It is onto because $\mathfrak{H}_{i+1}^{l}=\mathfrak{H}_{i}^{l}+\mathfrak{G}_{i+1}^{l}$ and its kernel is $\operatorname{Ker} \phi \simeq \frac{I_{l}^{(0)} \cap \mathfrak{G}_{i+1}^{l}}{I_{l}^{(0)} \cap \mathfrak{G}_{i}^{l}}$.

Consider the following natural map (multiplication)

$$
\rho_{i}^{l}: \frac{I_{i}^{(0)}}{I_{i}^{(0)} \cap W \cdot S^{i-1} T} \otimes S^{l-i} W \rightarrow \frac{I_{l}^{(0)} \cap \mathfrak{G}_{i+1}^{l}}{I_{l}^{(0)} \cap \mathfrak{G}_{i}^{l}} .
$$

Proposition 10. For any $0 \leq i \leq l$ the map $\varkappa_{i}^{l}$ of (2) is an isomorphism iff the map $\rho_{i}^{l}$ of (4) is an isomorphism.

Proof. Actually the isomorphism $\mathfrak{H}_{i+1}^{l} / \mathfrak{H}_{i}^{l} \simeq \tilde{g}_{i}^{*} \otimes S^{l-i} W$ due to (3) is equivalent to the isomorphism

$$
\operatorname{Ker} \phi \simeq\left(S^{i} V^{*} / \tilde{g}_{i}\right)^{*} \otimes S^{l-i} W \simeq\left(I_{i}^{(0)} / I_{i}^{(0)} \cap W \cdot S^{i-1} T\right) \otimes S^{l-i} W .
$$

Now let $V^{*}$ of dimension $r$ be non-characteristic and $W=\operatorname{ann}\left(V^{*}\right)$. Then the isomorphism property for $\rho_{i}^{l}$ and therefore Theorem $\mathrm{A}$ follow from

Proposition 11. $I_{l} \cap \mathfrak{G}_{i}^{l}=I_{i-1} \cdot S^{l-i+1} W$.

Proof. Actually, the statement follows inductively from the case $i=l: I_{l-1} \cdot W=$ $I_{l} \cap\left(W \cdot S^{l-1} T\right)$. If $I=\left\langle f_{1}, \ldots, f_{r}\right\rangle \subset S^{*} T$, this is equivalent to the following: for every element $\omega=\lambda_{1} f_{1}+\cdots+\lambda_{r} f_{r} \in I_{l} \cap\left(W \cdot S^{l-1} T\right)$ there exists another representation $\omega=\tilde{\lambda}_{1} f_{1}+\cdots+\tilde{\lambda}_{r} f_{r}$ with $\tilde{\lambda}_{j} \in W \cdot S^{l-d_{j}-1} T$.

Denote by $h^{0}$ the restriction of a polynomial $h$ on $T^{*}$ to $V^{*}$. Consider at first the case $r=2$. Because codim $\operatorname{Char}(g)=2$, the functions $f_{1}^{0}, f_{2}^{0}$ have no common divisor. So if $\omega=\lambda_{1} f_{1}+\lambda_{2} f_{2}$ satisfies $\omega^{0}=0$, then $\lambda_{1}^{0}=f_{2}^{0} h^{0}, \lambda_{2}^{0}=-f_{1}^{0} h^{0}$ and

$$
\tilde{\lambda}_{1}=\lambda_{1}-f_{2} h^{0}, \tilde{\lambda}_{2}=\lambda_{2}+f_{1} h^{0} \in W \cdot S T \text {. }
$$

Now let $r$ be arbitrary. For every subset $\sigma \subset S=\{1, \ldots, r\}$ define the ideals $I_{\sigma}=\left\langle f_{i} \mid i \in \sigma\right\rangle$. If $\omega^{0}=0$ we have $\lambda_{i}^{0} f_{i}^{0}=-\sum_{j \neq i} \lambda_{j}^{0} f_{j}^{0} \in I_{S \backslash\{i\}}$. Since $f_{i}^{0}$ has no divisor from this ideal (otherwise $\operatorname{Char}(g)$ has a smaller codimension), we deduce that $\lambda_{i}^{0}=\sum_{j \neq i} a_{i j}^{0} f_{j}^{0}$. Now $\omega^{0}=\sum_{i<j} \lambda_{i j}^{0} f_{i}^{0} f_{j}^{0}=0$, where $\lambda_{i j}^{0}=a_{i j}^{0}+a_{j i}^{0}$.

We can write this as $\lambda_{i j}^{0} f_{i}^{0} f_{j}^{0}=-\sum_{k<l}^{\prime} \lambda_{k l}^{0} f_{k}^{0} f_{l}^{0} \in I_{S \backslash\{i, j\}}$, where prime denotes all $(k, l) \neq(i, j)$. Since $f_{i}^{0} f_{j}^{0}$ has no divisor from this ideal, we get $\lambda_{i j}^{0}=$ $\sum_{k \neq i, j} a_{i j k}^{0} f_{k}^{0}$ and $\omega=\sum_{i<j<k} \lambda_{i j k}^{0} f_{i}^{0} f_{j}^{0} f_{k}^{0}$ with $\lambda_{i j k}^{0}=a_{i j k}^{0}+a_{i k j}^{0}+a_{j k i}^{0}$.

Continuing we get $\lambda_{\sigma}^{0}$ with multi-index of length $|\sigma|=r-1$. They would satisfy the property $\lambda_{S \backslash\{i\}}^{0}=a_{S \backslash\{i\}, i}^{0} f_{i}, \sum_{i=1}^{r} a_{S \backslash\{i\}, i}^{0}=0$. Now we can perform the 
required modification of the coefficients. For simplicity of notation we write it for the case $r=3$.

The change is: $\quad \tilde{\lambda}_{1}=\lambda_{1}-a_{12}^{0} f_{2}+a_{31}^{0} f_{3}-a_{132}^{0} f_{2} f_{3}$,

$$
\tilde{\lambda}_{2}=\lambda_{2}+a_{12}^{0} f_{1}-a_{23}^{0} f_{3}-a_{123}^{0} f_{3} f_{1}, \tilde{\lambda}_{3}=\lambda_{3}-a_{31}^{0} f_{1}+a_{23}^{0} f_{2}-a_{231}^{0} f_{1} f_{2} .
$$

Remark 8. Isomorphism (2) implies a non-canonical isomorphism $g_{l} \simeq \bigoplus \mathfrak{f}_{i}^{l} \simeq$ $\bigoplus_{i=0}^{l} \tilde{g}_{i} \otimes S^{l-i} W^{*}$. Thus we can calculate the dimension of $g_{l}$ using that of $\tilde{g}$ and $\operatorname{dim} W=n-r$. If $n-r=1$, then $\operatorname{dim} g_{l}=\sum_{0}^{l} \operatorname{dim} \tilde{g}_{i}$ stabilizes because $\tilde{g}$ is of finite type. On the other hand, if $n>r+1$, the dimension tends to infinity as $l$ grows: $\operatorname{dim} g_{l} \sim\left(\sum \operatorname{dim} \tilde{g}_{i}\right) \operatorname{dim} S^{l} W^{*}$. So we justify Remark 2 explicitly.

\section{Solvability CRITERION FOR PDEs OF $\operatorname{codim}(\mathcal{E})=2$}

3.1. Reduction of scalar PDEs. Consider a scalar PDEs system $\mathcal{E}$ of codim $=$ 2. It is given by two differential operators $F: J^{k}(M) \rightarrow \mathbb{R}, G: J^{l}(M) \rightarrow \mathbb{R}$. Suppose that these operators have independent symbols in all points of $\mathcal{E}$, i.e., the characteristic variety has codim $\operatorname{Char}(\mathcal{E})=2$. Then the ideal of the symbol $g$ is a complete intersection and so satisfies the hypothesis of Theorem A. Therefore for a non-characteristic two-dimensional subspace $V^{*}$ the reduction $\tilde{g}$ has the same Spencer cohomologies as the system $g$.

Moreover, suppose for at least one of the operators $F$ or $G$ that the characteristic variety has no multiple components, i.e., it possesses no defining equation of lower order. Let $\sigma(F)^{0}$ denote the restriction of symbol of the operator $F$ to $V^{*}$ and similarly for $G$. The reduced system $\tilde{g}$ on $V^{*}$ has no characteristics, i.e., the systems given by $\sigma(F)^{0}, \sigma(G)^{0}$ have no common characteristics, and at least one of them has distinct characteristics. So we can apply the results of KL2 for the computation of $H^{i, j}(g) \simeq H^{i, j}(\tilde{g})$ to conclude:

Proposition 12. Under the hypothesis of Theorem $\mathrm{B}$ at every point $x_{s}$ of the system $\mathcal{E}$ we have $H^{i, j}(\mathcal{E})=0$ for $j>2$. The only nonzero 1 st cohomologies have $\operatorname{dim} H^{i-1,1}(\mathcal{E})=1$ for $i=k, l$ if $k \neq l$, and $\operatorname{dim} H^{k-1,1}=2$ if $k=l$.

For the 2 nd cohomologies there is only one number $i=k+l-2$ such that $H^{i, 2}(\mathcal{E}) \neq 0$. Moreover, the bundle $H^{k+l-2,2}(\mathcal{E})$ is one-dimensional and the Weyl tensor $W_{k+l-1}(\mathcal{E})$ is its section (and so basis wherever nonzero).

We will show that this element $W_{k+l-1}(\mathcal{E})$ is the (scaled) Mayer bracket.

3.2. Higher Mayer brackets. Let $\left(x^{1}, \ldots, x^{n}\right)$ be a local coordinate system around $x \in M$. It produces a coordinate system $\left(x^{i}, p_{\sigma}\right)$ around a point $x_{k} \in J^{k}(M)$ with indices $1 \leq i \leq n$ and multi-indices $\sigma \in \mathbb{Z}_{\geq 0}^{n}, 0 \leq|\sigma| \leq k$. These coordinates produce a local isomorphism between $J^{k}(M)$ and $J^{k}\left(\mathbb{R}^{n}\right)$.

The total derivative operators $\mathcal{D}_{\sigma}: C^{\infty}\left(J^{l} M\right) \rightarrow C^{\infty}\left(J^{l+|\sigma|} M\right)$ for a multi-index $\sigma=\left(i_{1}, \ldots, i_{n}\right)$ are defined by $\mathcal{D}_{\sigma}=\mathcal{D}_{1}^{i_{1}} \ldots \mathcal{D}_{n}^{i_{n}}$, where $\mathcal{D}_{j}=\partial_{x^{j}}+p_{j} \partial_{u}+p_{\alpha j} \partial_{p_{\alpha}}+$ $\ldots$ is the total derivative by $x^{j}$.

Definition 5. The (higher) Mayer bracket of functions $F \in C^{\infty}\left(J^{k}\left(\mathbb{R}^{n}\right)\right)$ and $G \in C^{\infty}\left(J^{l}\left(\mathbb{R}^{n}\right)\right)$ is the following function on $J^{k+l}\left(\mathbb{R}^{n}\right)$ :

$$
[F, G]=\sum_{|\sigma|=l} \mathcal{D}_{\sigma}(F) G_{\sigma}-\sum_{|\tau|=k} \mathcal{D}_{\tau}(G) F_{\tau}, \quad \text { where } F_{\sigma}=\frac{\partial F}{\partial p_{\sigma}} .
$$


Thus we obtain a bracket of 2 scalar (non-linear) differential operators $F, G$ on $M$, which however depends on local coordinates, i.e., is not canonical. If in the above formula the summation is non-restricted, one gets the canonical well-known Jacobi bracket ([KLV] $)$. The following statement was proved in [KL2]:

Proposition 13. The Mayer bracket of a $k$-th order differential operator $F$ and an $l$-th order differential operator $G$ is a differential operator of the order $(k+l-1)$ : $[F, G] \in C^{\infty}\left(J^{k+l-1} M\right)$. The difference of this bracket and the Jacobi bracket $\{F, G\}$ belongs to the $(k+l-1)$-st order ideal generated by $F$ and $G$ :

$$
[F, G]-\{F, G\} \in \mathcal{J}^{(k, l)}(F, G)=\left\langle\mathcal{D}_{\sigma}(F), \mathcal{D}_{\tau}(G)\right\rangle_{0 \leq|\tau|<k, 0 \leq|\sigma|<l} .
$$

If $\mathcal{E}$ is the system defined by the equations $F=0, G=0$, we deduce that the restriction of the Mayer bracket $[F, G]$ to the prolongation $\mathcal{E}_{k+l-1}$ (that always exists!) does not depend on a choice of local coordinates and so is canonical.

3.3. Proof of Theorem B, Note that every non-characteristic subspace $V^{*} \subset T^{*}$ of dimension 2 induces a monomorphism of the complex $\tilde{g} \otimes \Lambda V^{*}$ into $g \otimes \Lambda T^{*}$. This in turn induces a map of cohomologies which is an isomorphism due to the spectral sequences considerations of 2.2 2.4. Thus the results of [KL2] imply the isomorphism of the following 1-dimensional spaces: $H^{k+l-2,2}(g) \simeq \tilde{g}_{k+l-2} \otimes \Lambda^{2} V^{*}$.

Now due to Proposition 12 and the integrability criterion of $[\mathrm{Ly}]$ to prove Theorem $\mathrm{B}$ it is sufficient to prove

Theorem 14. The only nonzero Weyl tensor of the PDEs system $\mathcal{E}=\{F=$ $0, G=0\}$ is $W_{k+l-1}(\mathcal{E})=\left.[F, G]\right|_{\mathcal{E}_{k+l-1}} \cdot[\omega]$, where the class $[\omega]$ is a basis element of $H^{k+l-2,2}(\mathcal{E})$. Moreover, given a non-characteristic two-dimensional subspace $V^{*} a$ representative $\omega$ can be chosen as the monomorphic image of $\tilde{\omega}=\frac{e_{\tilde{g}}}{R\left(\sigma_{F}, \sigma_{G}\right)} \otimes d x^{1} \wedge$ $d x^{2}$, where $d x^{1}, d x^{2}$ is a basis of $V^{*}, R\left(\sigma_{F}, \sigma_{G}\right)$ is the resultant of the symbols of $F$ and $G$ restricted to $V^{*}$ and the element

$$
e_{\tilde{g}}=\operatorname{det}\left\|\begin{array}{cccccccc}
F_{k, 0} & F_{k-1,1} & \ldots & \ldots & F_{0, k} & 0 & \ldots & 0 \\
0 & F_{k, 0} & \ddots & & F_{1, k-1} & F_{0, k} & & \vdots \\
\vdots & \vdots & \ddots & & \ldots & F_{1, k-1} & \ddots & 0 \\
0 & 0 & \ldots & F_{k, 0} & \ldots & & \ldots & F_{0, k} \\
G_{l, 0} & G_{l-1,1} & \ldots & G_{2, l-2} & \ldots & & \ldots & 0 \\
0 & G_{l, 0} & \ddots & \vdots & \ddots & G_{0, l} & \ddots & 0 \\
\vdots & \ldots & \ddots & & \ddots & & \ddots & 0 \\
0 & 0 & \ldots & G_{k, l-k} & \ldots & & \ldots & G_{0, l} \\
e_{k+l-2,0} & e_{k+l-3,1} & \ldots & & e_{l-2, k} & \ldots & & e_{0, k+l-2}
\end{array}\right\|
$$

is a basis element of $\tilde{g}_{k+l-2} \subset S^{k+l-2} V^{*}, e_{i, k-i}=\frac{\left(d x^{1}\right)^{i}\left(d x^{2}\right)^{k-i}}{i !(k-i) !}$. By the subscript indices in $F_{i, j}, G_{i, j}$ we mean the differentiation by $p_{\sigma}=p_{i, j}$, where $\sigma=1_{1}^{i}+1_{2}^{j}$ (the multi-index with $i$ number of 1 and $j$ number of 2).

Proof. Recall ( 1.4 and $\left[\mathrm{Ly}\right.$ ) that the Weyl tensor $W_{k+l-1}(\mathcal{E})$ of a system $\mathcal{E}$ is the $\delta$-cohomology class of the metasymplectic structure restriction $\left.\left(\Omega_{k+l-1}\right)\right|_{H}$, where $H=\left\langle\nabla_{1}, \ldots, \nabla_{n}\right\rangle$ is the horizontal subspace generated by $\nabla_{i}=\mathcal{D}_{i}^{(k+l-1)}+$ 
$\sum_{|\sigma|=k+l-1} \alpha_{i}^{\sigma} \partial_{\sigma}$ and $\mathcal{D}_{i}^{(s)}$ is the total derivative restricted to $J^{s}(M)$. The metasymplectic form has in coordinates the following form ( KL1 $)$ :

$$
\Omega_{s}=\sum_{|\sigma|=s-1} \partial_{p_{\sigma}} \otimes d \omega_{\sigma}, \quad \omega_{\sigma}=d p_{\sigma}-\sum_{j} p_{\sigma+1_{j}} d x^{j} .
$$

We claim that there exists a horizontal space $H$ such that

$$
\left.\left(\Omega_{k+l-1}\right)\right|_{H} \in \tilde{g}_{k+l-2} \otimes \Lambda^{2} V^{*} \subset g_{k+l-2} \otimes \Lambda^{2} T^{*} .
$$

In fact, the inclusion of complexes $\tilde{g}_{i} \otimes \Lambda^{j} V^{*} \subset g_{i} \otimes \Lambda^{j} T^{*}$ induces an isomorphism of cohomologies and so a representative for $W_{k+l-1}$ can be chosen in the required form. The specified space $H$ is not unique, but because $\tilde{g}_{k+l-1}=0$ (see [KL1]), the restriction of $\Omega_{k+l-1}$ to it is canonical. Therefore, for each such $H$ we have

$$
\left.\left(\Omega_{k+l-1}\right)\right|_{H}=\sum_{\sigma=1_{1}^{i}+1_{2}^{j}} d \omega_{\sigma}\left(\nabla_{1}, \nabla_{2}\right) \partial_{\sigma} \otimes d x^{1} \wedge d x^{2} ; \quad i+j=k+l-2 .
$$

Let us evaluate $d \omega_{\sigma}\left(\nabla_{1}, \nabla_{2}\right)$. The condition $H \subset T \mathcal{E}_{k+l-1}$ is equivalent to the following system:

$$
\left\{\begin{array}{lll}
\sum_{\left|\tau^{\prime}\right|=k} \alpha_{i}^{\sigma^{\prime}+\tau^{\prime}} F_{\tau^{\prime}}=-\mathcal{D}_{\sigma^{\prime}+1 i}^{(k+l-1)} F, & \left|\sigma^{\prime}\right|=l-1, \quad 1 \leq i \leq n, \\
\sum_{\left|\tau^{\prime \prime}\right|=l} \alpha_{i}^{\sigma^{\prime \prime}+\tau^{\prime \prime}} G_{\tau^{\prime \prime}}=-\mathcal{D}_{\sigma^{\prime \prime}+1_{i}}^{(k+l-1)} G, & \left|\sigma^{\prime \prime}\right|=k-1 .
\end{array}\right.
$$

Coefficients $\alpha_{i}^{\varkappa}$ are symmetric in multi-indices $\varkappa$. Condition (6) is equivalent to the symmetry of $\hat{\alpha}^{\hat{\varkappa}}:=\alpha_{i}^{\varkappa}$ by $\hat{\varkappa}=i \varkappa=\varkappa+1_{i}$ whenever this multi-index contains at least one index $j \neq 1,2$.

Thus we have an underdetermined linear system with $\varrho_{k}^{n}+\varrho_{l}^{n}$ equations and $\varrho_{k+l}^{n}$ unknowns, where $\varrho_{r}^{n}=\left(\begin{array}{c}n+r-1 \\ r\end{array}\right)+(r-1)$. Whenever the symbols of $F$ and $G$ are independent (as in the assumptions of the theorem), it has the full rank and so is compatible. The values

$$
d \omega_{\sigma}\left(\nabla_{1}, \nabla_{2}\right)=\alpha_{2}^{\sigma+1_{1}}-\alpha_{1}^{\sigma+1_{2}}, \quad \sigma=1_{1}^{i}+1_{2}^{j}, i+j=k+l-2
$$

do not depend on the choice of a solution $\hat{\alpha}^{\hat{\varkappa}}$. It follows from (7) that they are proportional to the Mayer brackets as indicated in the statement.

Let us illustrate the above proof using the example $k=l=2$ (for $n=2$ it was studied in detail in [KL2]). System (77) has $n^{2}+n+2$ equations and $\frac{1}{24} n$ $(n+1)(n+2)(n+3)+3$ unknowns. It is solvable and we calculate:

$$
d \omega_{\sigma}\left(\nabla_{1}, \nabla_{2}\right)=\alpha_{2}^{\sigma 1}-\alpha_{1}^{\sigma 2}=\frac{[F, G]}{\varpi} d p_{\sigma} \wedge d^{v} F \wedge d^{v} G\left(\partial_{p_{11}}, \partial_{p_{12}}, \partial_{p_{22}}\right),
$$

$\sigma \in\{11,12,22\}$, where $\varpi=\rho_{12}^{2}-\rho_{11} \rho_{22}, \rho_{11}=F_{12} G_{22}-F_{22} G_{12}, \rho_{12}=F_{22} G_{11}-$ $F_{11} G_{22}, \rho_{22}=F_{12} G_{12}-F_{12} G_{11}$ and $F_{i j}=\partial_{p_{i j}} F, G_{i j}=\partial_{p_{i j}} G$. Thus

$$
W_{3}=\frac{e_{\tilde{g}}}{\varpi}[F, G]_{\mathcal{E}} \otimes d x^{1} \wedge d x^{2}, \quad e_{\tilde{g}}=\rho_{11} \partial_{p_{11}}+\rho_{12} \partial_{p_{12}}+\rho_{22} \partial_{p_{22}} .
$$

3.4. Miscellaneous results. 1. Solutions space. Let the scalar system $\mathcal{E}=\{F=$ $0, G=0\}$ satisfy the hypotheses of Theorem B. Denote by $\mathcal{R}_{\mathcal{E}}$ the space of (germs of) its solutions. It is finite-dimensional if and only if $\operatorname{dim} M=2$. Suppose $\operatorname{ord}(\mathcal{E})=$ $\{k, l\}$ and $k \leq l$.

Proposition 15. If $\operatorname{dim} M=2$, then $\mathcal{R}_{\mathcal{E}}$ is smooth and $\operatorname{dim} \mathcal{R}_{\mathcal{E}}=k l$. 
Proof. Since the considered case is of finite type, Frobenius theorem applied to $\mathcal{E}_{\infty} \simeq \mathcal{E}_{k+l-1}$ implies smoothness. The dimension formula follows from the calculation of $\operatorname{dim} g_{i}$ in Proposition 13 KL2]:

$\operatorname{dim} \mathcal{R}_{\mathcal{E}}=\operatorname{dim} \mathcal{E}_{\infty}-2=\sum_{i=0}^{\infty} \operatorname{dim} g_{i}=\sum_{i=0}^{k-1}(i+1)+\sum_{i=k}^{l-1} k+\sum_{i=l}^{k+l-1}(k+l-1-i)=k l$.

2. Lax-pairs. Consider a pair of scalar linear evolution operators $F=\partial_{t}+A$ and $G=\partial_{t}+B$ on the space $\mathbb{R}^{1} \times M^{n}$, where $A=\sum a_{\sigma}(t, x) \partial_{x}^{\sigma}$ and $B=\sum b_{\sigma}(t, x) \partial_{x}^{\sigma}$ are $t$-dependent linear differential operators on $M(t \in \mathbb{R}, x \in M)$.

Since for linear operators $F$ and $G$ the Jacobi bracket $\{F, G\}$ coincides with the usual commutator, the Mayer bracket $[F, G]$ equals the commutator modulo the ideal $\mathcal{J}^{(k, l)}(F, G)$ from Proposition 13,

Proposition 16. If the symbols of the linear operators $A$ and $B$ are independent, then the system $\mathcal{E}=\{F=0, G=0\}$ is compatible iff the commutator $[A, B]=\nabla(A-B)$ for some linear differential operator $\nabla$ on $\mathbb{R} \times M$ of $\operatorname{ord}(\nabla)<$ $\max \{\operatorname{ord}(A), \operatorname{ord}(B)\}$.

Proof. Due to Theorem $\mathrm{B}$ the compatibility of $\mathcal{E}$ is given by the condition $\left[\partial_{t}+\right.$ $\left.A, \partial_{t}+B\right]=\alpha \circ\left(\partial_{t}+A\right)+\beta \circ\left(\partial_{t}+B\right), \operatorname{ord}(\alpha)<\operatorname{ord}(B), \operatorname{ord}(\beta)<\operatorname{ord}(A)$, where by [,] one can understand both the Mayer bracket and the commutator. The last condition is clearly equivalent to the condition in the statement. Note that for $n=1$ and $\operatorname{ord}(F), \operatorname{ord}(G)>1$ the symbols become dependent, so that Theorem B cannot be applied directly, but an easy modification with a higher weight for the $t$-variable solves the problem.

In particular, if $\nabla=\partial_{t}$, we get the standard commutativity condition for Laxpairs: $[A, B]=\partial_{t} A-\partial_{t} B$.

3. Integrability of characteristics. Consider a system $\mathcal{E}=\left\{F_{1}=0, \ldots, F_{m}=0\right\}$ of scalar linear PDEs of $\operatorname{codim}(\mathcal{E})=m$ on a manifold $M^{n}$. The compatibility condition implies vanishing of the Mayer bracket $\left[F_{i}, F_{j}\right]$ due to the system. For $m>n$ these are the conditions of orders higher than the orders of the actual compatibility conditions (cf. [KL2], §3.2). But in some cases, when $m \leq n$, these conditions are equivalent to the compatibility.

We can reformulate them by saying that the ideal generated by $F_{1}, \ldots, F_{m}$ is closed under the Jacobi-Mayer bracket in a sense that addition of these brackets does not change the formal codimension of the corresponding equation $\mathcal{E}$. Since the symbol of the Jacobi bracket is the Poisson structure on the algebra of symbols, we deduce (for systems $\mathcal{E}$ of the kind specified above) the integrability of characteristics condition ([GQS]): the (radical of) charateristic ideal is Poisson.

\section{Applications}

4.1. Auxiliary integrals. Consider a (non-linear) formally integrable (=compatible) system $\mathcal{E} \subset J^{k}(\pi)$.

Definition 6. Call a compatible system $\tilde{\mathcal{E}}$ an auxiliary integral (or a set of integrals) for the system $\mathcal{E}$ if the joint system $\mathcal{E} \cap \tilde{\mathcal{E}}$ is compatible too.

Consider the particular case of codimension one scalar PDEs $\mathcal{E}=\{F=0\}$, $\tilde{\mathcal{E}}=\{G=0\}$. Then $G$ is an auxiliary integral iff $[F, G]_{\mathcal{E}}=0$. Equivalently we can 
write $\{F, G\}=P \circ F+Q \circ G$, where $P$ and $Q$ are differential operators of orders $l-1$ and $k-1$ respectively.

Recall ([KLV] $)$ that a symmetry of a system $\mathcal{E}=\bigcap_{i=1}^{r}\left\{F_{i}=0\right\}$ is such a differential operator $G$ that $\left\{F_{i}, G\right\}=\sum \lambda_{i}^{j} \circ F_{j}, i=1, \ldots, r$, for some differential operators $\lambda_{i}^{j}$. If $G$ is of the first order, it is called a classical symmetry (a point transformation if lifted from a field on $J^{0}(\pi)$ and a contact symmetry if a general field on $J^{1}(\pi)$ ) and it is called a higher symmetry otherwise.

An equation $\tilde{\mathcal{E}} \subset J^{s}(\pi)$ is an intermediate integral for an equation $\mathcal{E} \subset J^{t}(\pi)$ if $s<t$ and the prolongation $\tilde{\mathcal{E}}^{(t-s)} \subset \mathcal{E}$. In other words, every solution of the $\operatorname{PDE} \tilde{\mathcal{E}}$ is a solution to the system $\mathcal{E}$. Using this rephrasing, let us say that $\tilde{\mathcal{E}}$ is an auxiliary integral if some solutions of the PDE $\tilde{\mathcal{E}}$ are solutions to the system $\mathcal{E}$ in the sense that the two equations are compatible.

Proposition 17. (i) Intermediate integrals are auxiliary integrals.

(ii) For one scalar PDE $F=0$ the classical and higher symmetries are auxiliary integrals also, whenever the characteristic varieties of the PDE and the symmetry are transversal.

Proof. The first statement is obvious. The second one follows from Theorem B, If $G$ is a symmetry, then the Mayer bracket $[F, G]_{\mathcal{E}}$ vanish.

Remark 9. The second statement holds also in a more general context, when $\tilde{\mathcal{E}}$ is a subalgebra of the symmetry algebra $\operatorname{Sym}(\mathcal{E})$ with arbitrary codimension of $\mathcal{E} \subset J^{k}(\pi)$, if certain transversality conditions are imposed.

Traditional methods of solving PDEs are based on symmetries, intermediate integrals and similar tools (Gou $)$. The Lagrange-Charpit method consists of finding an overdetermination of a special form for a given PDE to solve it.

Auxiliary integrals can also be used for the same purpose. Namely the additional equation being solved, determines an ansatz, substitution of which reduces/solves the original PDE. We will call this procedure the generalized Lagrange-Charpit method.

Remark 10. The known method of differential constraints does not coincide with the proposed method of auxiliary integral. For we can impose a differential constraint so that the obtained system is not compatible, but deriving other differential corollaries we arrive at a compatible system.

Thus the auxiliary integrals lead to compatible systems, while for differential constraints one gets solvable systems. Note though that the Mayer bracket can be used for differential constraints as well: If it is not zero, it should be added to the system as its differential corollary.

Let us sketch an algorithm to search for overdeterminations for one linear scalar PDE $F=0$. Denote the symbol of an operator $H$ by $\sigma(H)$. If $G$ is an auxiliary integral for $F$, we have an identity for the symbols

$$
\{\sigma(F), \sigma(G)\}=\lambda_{1} \cdot \sigma(F)+\lambda_{2} \cdot \sigma(G),
$$

where $\{$,$\} is the Poisson bracket here. Thus the ideal \langle\sigma(F), \sigma(G)\rangle$ is Poisson. Given $F$ such ideals can be seen as solutions of the Hamilton-Jacobi equation.

So we start by solving symbolic equation (8). In the case of dimension $n=2$ the problem can always be solved: Any $\sigma(G)$ works, whenever it has no common 
characteristics with $\sigma(F)$. For $n>2$ there are obstructions, as a simple parameter count shows.

Remark 11. Consider a complex linear operator $P=F+i G, F=\operatorname{Re}(P), G=$ $\operatorname{Im}(P)$, over a real base manifold $M$. A necessary condition for local solvability (existence of a solution) of the differential equation $P(u)=g$ for an arbitrary smooth complex function $g$ ( $u$ is also complex-valued) is the following Hörmander's condition ([을 $)$ : the ideal generated by $\sigma(F)$ and $\sigma(G)$ must be Poisson (or equivalently $\left.\sigma\left([F, G]_{\mathcal{E}}\right)=0\right)$. Thus our condition appears in other solvability problems.

Next we take some $G_{1}$ with the obtained symbol $\sigma(G)$. We write $G=G_{1}+G_{2}^{\prime}$, with the order of $G_{2}^{\prime}$ less by 1 than that of $G_{1}$, and write the equation for the symbol $\sigma\left(G_{2}^{\prime}\right)$, arising from the compatibility condition $[F, G]_{\mathcal{E}}=0$. Solving it we continue: Let $G=G_{2}+G_{3}^{\prime}$, study the compatibility and so on. This inductive procedure stops in a finite number of steps.

We remark that usually the symbolic equations form an overdetermined system for the coefficients of $F$ and the compatibility gives restrictions for the existence of auxiliary integrals of a given $F$. For $n=2$ this scheme produces some explicit restrictions on $F$. Details will be considered elsewhere.

4.2. Associativity equation. This equation also named as the Witten-DijkgraafH. Verlinde-E. Verlinde (WDVV) equation arises in the topological field theory and is basic for Frobenius manifolds and quantum cohomology Man. We consider this equation in dimension 3 , i.e., on $\mathbb{R}^{3}\left(x^{0}, x^{1}, x^{2}\right)$. It is an equation on one function $u=u\left(x^{1}, x^{2}\right)$ and it has the form:

$$
F=p_{222}-p_{112}^{2}+p_{111} p_{122}=0 .
$$

In [D] Dubrovin find solutions via the scaling symmetries: $G=s x^{0} p_{0}+\lambda x^{1} p_{1}+$ $\mu x^{2} p_{2}+c u$, where to cover all affine combinations we adopt the following convention: $\left.\lambda \cdot x^{1}\right|_{\lambda=0}=\tilde{\lambda}$ and similarly for $s x^{0}, \mu x^{2}$ and $c u$. We will drop the variable $x^{0}$ in the sequel. This is irrelevant for symmetries, but an auxiliary integral $G$, which is not a symmetry, has inevitably $s=0$. We have

$$
[F, G]=(4 \lambda+2 \mu+c) F+(\mu-4 \lambda-c) p_{222} .
$$

So $G=0$ is a symmetry for (9) iff $c=\mu-4 \lambda$. In topological field theory the following particular case is of importance: $\lambda=-D / 2, \mu=1-D, c=1+D$.

We will drop the quasi-homogeneity assumption for the solutions of the WDVV, which is the requirement of a scaling symmetry. Below we will also write $x=x^{1}$, $y=x^{2}$.

1. First order auxiliary integrals. Using the calculation with the Mayer bracket (5) and Theorem B we get that function $G=\lambda x p_{1}+\mu y p_{2}+c u$ (with the above convention) is an auxiliary integral of the associativity equation (9) iff it is one of the following:

A) $G=\lambda x p_{1}+\mu y p_{2}+(\mu-4 \lambda) u$;

B) $G=x p_{1}+y p_{2}-2 u ; G=x p_{1}-u ; G=x p_{1}+\tilde{c} ; G=u$;

C) $G=y p_{2}-r u, r=1,2$.

The proof is obtained by the direct reduction of the term $p_{222}$ in (10) modulo the functions $G, \mathcal{D}_{i} G, \mathcal{D}_{i j} G, F$. 
The symmetries A) reduce (9) to the Painelve-VI equation ([D]). The other cases are not symmetries: $\mathrm{B}$ ) and $\mathrm{C})_{1}$ give trivial solutions (quadratic or functions of one variable). The case $\mathrm{C})_{r=2}$ gives the solution $u=y^{2} \ln x$.

This last is the only (modulo trivial) solution of the form $f(x) g(y)$, which is not obtained from the other symmetries. In fact, the scaling symmetry A) for $\mu=0$ gives $u=\frac{1}{16} x^{4} g(y)$, where $g(y)$ satisfies the ODE $g^{\prime \prime \prime}-9\left(g^{\prime}\right)^{2}+6 g g^{\prime \prime}=0$; and the scaling symmetry A) for $\lambda=0$ gives $u=f(x) / y$, where $f(x)$ satisfies the ODE $2 f^{\prime \prime \prime} f^{\prime}-\left(f^{\prime \prime}\right)^{2}-6 f=0$ (this last equation was reduced by Dubrovin to the Painlevé transcendents). The two families of solutions have only two intersections: $u=0$ and $u=\frac{1}{8} x^{4} / y$.

2. Higher order auxiliary integrals. Another choice of overdetermination is given by the higher order linear operators with constant coefficients

$$
G=a p_{11}+b p_{12}+c p_{22} \quad \text { and } \quad G=a p_{111}+b p_{112}+c p_{122}+d p_{222} .
$$

They satisfy the compatibility $[F, G]_{\mathcal{E}}=0$, but are not higher symmetries.

Due to Proposition [15] for any $a, b, c$ in the first choice of $G$ the system $\mathcal{E}$ has a 6-parametric solution, of which a 5 -parametric part is trivial. Similarly, for any $a, b, c, d$ in the second choice of $G$ the system $\mathcal{E}$ has a 9 -parametric solution, of which an 8-parametric part is trivial.

Other auxiliary integrals are $G=p_{1111}, G=p_{11111}$ and they lead to the solutions of the WDVV related to the symmetries of Platonic solids $[\mathrm{D}$.

Let us note that the ansatz $u=f(x) g(y)$ cannot be covered by a second order auxiliary integral (like $G=p_{12} u-p_{1} p_{2}$, which is through a differential constraint) because due to Proposition 15 the space of such solutions would be 6-dimensional, while the exact calculations show it has dimension 3 . In fact, in subsection $\mathbf{1}$ above we covered the ansatz by three different auxiliary integrals.

3. Symmetric auxiliary integrals. Now we consider a symmetric overdetermination. In this way we obtain the following non-trivial higher auxiliary integrals for PDE (9):

A) $G=2 p_{11} p_{22}-3 p_{12}^{2}$;

B) $G=2 p_{112}-p_{111}^{2}$.

To explain this we remark that equation (9) has a 12-dimensional Lie algebra $\mathcal{G}$ of point symmetries. We describe its basis via the generating ([KLV]; in other sources they are named characteristic) functions:

$$
\begin{gathered}
e_{1}=1, e_{2}=x, e_{3}=y, e_{4}=x^{2}, e_{5}=x y, e_{6}=y^{2}, e_{7}=p_{1}, e_{8}=p_{2}, \\
e_{9}=x p_{1}-4 u, e_{10}=y p_{2}+u, e_{11}=y p_{1}-x^{3} / 2, e_{12}=y x p_{1}+y^{2} p_{2}-x^{4} / 8-2 y u
\end{gathered}
$$

It has two subalgebras: $\mathcal{G}_{A}=\left\langle e_{1-3}, e_{7-10}\right\rangle$ and $\mathcal{G}_{B}=\left\langle e_{1-10}\right\rangle$ of dimensions 7 and 10 respectively. They consist of symmetries for the cases A) and B), where the prolonged equation $\mathcal{E}_{\infty}$ has dimension 8 or 10 correspondingly.

Since both $\mathcal{G}_{A}$ and $\mathcal{G}_{B}$ are reductive (of the length 3 ), the system $\mathcal{E}=\{F=$ $0, G=0\}$ can be solved in quadratures by the Lie method.

Let us find solutions of the WDVV via the auxiliary integral $G$ of B). By the triple differentiation, substitution and simplification we bring (9) to the ODE

$$
f^{\prime \prime \prime} f^{\prime} f=2 f^{\prime \prime}\left(f^{\prime}\right)^{2}+3\left(f^{\prime \prime}\right)^{2} f,
$$


where $f=p_{111}$ and prime means the differentiation by $x$. One can check the following compatibility: every solution to the above equation with $f^{\prime \prime} \neq 0$ yields a solution of the WDVV (the case $f^{\prime \prime}=0$ was considered in subsection 2 above).

The ODE admits complete integration: $A(y) f+B(y) f^{4}+x+C(y)=0$. This equation with $f=v_{x}$ should be compatible with $v_{x}^{2}=2 v_{y}\left(v=u_{x x}\right)$ and this implies: $A(y)=y+a, B(y)=b, C(y)=c$. Let $f=w(x, y)$ be the solution to the equation $(y+a) f+b f^{4}+(x+c)=0 . f^{\prime \prime} \neq 0$ is equivalent to $b \neq 0$.

Finally, we obtain the system

$$
\begin{cases}u_{x x x} & =w(x, y), \\ u_{x x y} & =w^{2}(x, y) / 2, \\ u_{y y y}+w(x, y) u_{x y y} & =w^{4}(x, y) / 4,\end{cases}
$$

which is exactly solvable in quadratures.

Remark 12. Since the solution space of the equation $\mathcal{E}=\{F=0, G=0\}$ in the case B) has $\operatorname{dim} \mathcal{R}_{\mathcal{E}}=9$ (Proposition 15), but $\operatorname{dim} \mathcal{G}_{B}=10$, every solution found in this approach can be also obtained via a symmetry. Note, however, that some symmetries from $\mathcal{G}_{B}$ lead to transcendents, while we got all our solutions in quadratures.

4.3. Linear second order PDEs and projective geometry. Consider a system of scalar linear PDEs $\mathcal{E} \subset J^{2}\left(M^{2}\right)$ of $\operatorname{codim} \mathcal{E}=2$. Whenever formally integrable, it is smoothly integrable and the linear space of solutions $\mathcal{R}_{\mathcal{E}}$ is 4-dimensional. In fact, the Cartan distribution on the 6 -dimensional $\mathcal{E}^{(1)} \simeq \mathcal{E}$ is a rank 2 integrable distribution (Cartan connection).

In what follows we work locally. A milder restriction is possible: We assume that $\pi_{1}(M)$ is trivial or that the Cartan connection on $\mathcal{E}$ has no holonomy.

Consider the equivalence of such PDEs $\mathcal{E}$ with respect to the pseudogroup of transformations induced from automorphisms of the vector bundle $J^{0}(M)=M \times \mathbb{R}$. The following statement is essentially due to Wilczynski [W]:

Theorem 18. Equivalence classes of compatible scalar systems $\mathcal{E} \subset J^{2}\left(M^{2}\right)$ of finite type and $\operatorname{codim} \mathcal{E}=2$ correspond bijectively to the classes of projectively equivalent surfaces $\hat{M}^{2} \subset \mathbb{P}^{3}$.

Proof. Let $\mathcal{E}$ be a compatible system. Then it is integrable and has a basis of local solutions $r_{0}, r_{1}, r_{2}, r_{3}$. Since this is a basis of a linear system, the vector-valued function $\mathbf{r}=\left(r_{0}, r_{1}, r_{2}, r_{3}\right)$ never vanishes. So its projectivization, given by the position vector $\left[r_{0}: r_{1}: r_{2}: r_{3}\right]$, defines a surface $\hat{M}^{2}(x, y) \subset \mathbb{P}^{3}$. Change of a solutions basis $\left\{r_{i}\right\}_{i=0}^{3}$ leads to a projective transformation of $\hat{M}$.

Conversely, let functions $\left(r_{0}, r_{1}, r_{2}, r_{3}\right)$ define an affine surface with the projectivization $\hat{M}$. For each $(x, y) \in \hat{M}$ the 6 -dimensional vectors $j_{(x, y)}^{2} r_{i}, 1 \leq i \leq 4$, obey 2 linear relations $F\left(j^{2} r\right)=0, G\left(j^{2} r\right)=0$. The equation $\mathcal{E}$ is now $\{F=G=$ $0\}$.

A projective equivalence of surfaces corresponds to an isomorphism of linear equations $\mathcal{E}$ given by a change of dependent and independent variables.

This result leads to an important interplay between PDEs and projective differential geometry (cf. [W], [F]). For example, the symbol $g \subset S^{2} T^{*} M$ of the system $\mathcal{E}$ is one-dimensional and its sections are quadrics on $M$. Thus a PDE $\mathcal{E}$ defines a $g^{*}$-valued quadric $q_{\mathcal{E}}$ on $M$, which can also be considered as a conformal quadric. 
It corresponds to the second fundamental form in projective geometry, which is a quadric on $T \hat{M}$ with the values in the 1-dimensional bundle $T \mathbb{P}^{3} / T \hat{M}$. Elliptic, hyperbolic and parabolic points on $\hat{M}^{2}$ correspond to the points on $M^{2}$, where the quadric $q_{\mathcal{E}}$ has the same property.

At non-degenerate points the conformal quadric $q_{\mathcal{E}}$ can be brought to its wellknown normal forms used below. Note that there's no distinction between hyperbolic and elliptic cases over $\mathbb{C}$. We will consider the situation over $\mathbb{R}$.

For non-characteristic systems $\mathcal{E}$ (elliptic or hyperbolic), considered in the above theorem, the compatibility condition is expressed via the Mayer bracket: $[F, G]_{\mathcal{E}}=$ 0 .

1. Hyperbolic systems. For hyperbolic systems the null-directions of $q_{\mathcal{E}}$ are called asymptotic lines for the corresponding $\hat{M} \subset \mathbb{P}^{3}$. Since the quadric can be brought to the form $q_{\mathcal{E}}=d x d y$ ( $\{x=$ const, $y=$ const $\}$ are asymptotic lines $)$, the system $\mathcal{E}$ can be defined by the equations

$$
F=r_{x x}+\phi\left(r_{x}, r_{y}, r\right), \quad G=r_{y y}+\psi\left(r_{x}, r_{y}, r\right)
$$

with linear functions $\phi=a_{1} r_{x}+b_{1} r_{y}+c_{1} r, \psi=a_{2} r_{x}+b_{2} r_{y}+c_{2} r$.

The compatibility condition $[F, G]_{\mathcal{E}}=0$ consists of 4 equations on 6 functions $a_{i}, b_{i}, c_{i}$, and the equation $\left(a_{1}\right)_{y}=\left(b_{2}\right)_{x}$ is among them. So following Wilczynski [W] we transform the equation by a rescaling $r \mapsto \rho \cdot r$ to the form:

$$
F=r_{x x}-\beta r_{y}-\lambda r=0, G=r_{y y}-\gamma r_{x}-\mu r=0, \quad \beta, \gamma, \lambda, \mu \in C^{\infty}\left(M^{2}\right) .
$$

Compatibility of this new $\mathcal{E}$ gives 3 equations on 4 functions $\beta, \gamma, \lambda, \mu$, corresponding to the coefficients of $r_{x}, r_{y}, r$ in $[F, G]_{\mathcal{E}}$ :

$$
\begin{gathered}
\gamma_{x x}=2 \mu_{x}-2 \gamma \beta_{y}-\beta \gamma_{y}, \beta_{y y}=2 \beta \gamma_{x}+\gamma \beta_{x}-2 \lambda_{y}, \\
\mu_{x x}-\lambda_{y y}=\beta \mu_{y}-\gamma \lambda_{x}+2 \mu \beta_{y}-2 \lambda \gamma_{x} .
\end{gathered}
$$

Using the generalized Lagrange-Charpit method (or the common idea of ansatz), as proposed in KL2] and \$4.1, we will deduce several compatible cases describing the known surfaces.

A) Let $\beta=\gamma=0, \lambda=\lambda(x), \mu=\mu(y)$. Then $[F, G]_{\mathcal{E}}=0$ and the system (11) is compatible. The corresponding surfaces are quadrics.

B) Let us add to underdetermined system (12) the equation $\beta=\gamma$. We obtain a $3 \times 3$ formally integrable determined system. To reduce it to a scalar PDE, we overdetermine it via the following ansatz ( $s, t, a$ are constants):

$$
\lambda=s(\ln \beta)_{x x}+t(\ln \beta)_{x}^{2}+a \beta_{y}, \mu=s(\ln \beta)_{y y}+t(\ln \beta)_{y}^{2}+a \beta_{x} .
$$

So we get a system $\mathcal{E} \subset J^{3}\left(\mathbb{R}^{2}\right)$ of $\operatorname{codim}(\mathcal{E})=3$. One easily computes that $H^{2,2}(\mathcal{E})$ is one-dimensional and the only compatibility condition is $(2 a+1)\left(\beta_{x} \beta_{x x}-\right.$ $\left.\beta_{y} \beta_{y y}\right)=0$. If $a=-\frac{1}{2}$, the system is compatible. If not we add the equation $\beta_{x} \beta_{x x}=\beta_{y} \beta_{y y}$ to the system and study it again by the prolongation-projection method. Finally, we arrive at the following second-order system:

$$
\beta_{x x}=2 b \beta \beta_{y}, \beta_{x y}=\frac{\beta_{x} \beta_{y}}{\beta}+b^{2} \beta^{3}, \beta_{y y}=2 b \beta \beta_{x},
$$

(the second is the Liouville equation) and $b$ can be found from the equation

$$
3-4 b^{2}(s+t)= \pm 2 b(1+2 a) .
$$


Thus we have proved the following statement:

The considered ansatz reduces the compatibility system (12) to the Frobenius type system (13), which can be integrated in quadratures.

Several particular cases when the condition is fulfilled are well-known. Namely for $a=-7 / 4, s=-1 / 4, t=1 / 16, b=4 / 9$ we get Roman surfaces of Steiner. The case $a=3 / 4, s=-1 / 4, t=1 / 16, b=4 / 9$ corresponds to cubic surfaces, which are in fact dual to the above Steiner quartics. The special compatible case $a=-1 / 2$ leads to the quartics of Kummer if we overdetermine the system once more by the Liouville equation $(\ln \beta)_{x y}=b \beta^{2}$.

C) Consider an overdetermination of system (12) by the equation $\beta_{x}=\gamma_{y}$, i.e., $\beta=\varphi_{y}, \gamma=\varphi_{x}$. We reduce it to a scalar PDE via the following ansatz:

$$
\lambda=a \varphi_{x}^{2}+b \varphi_{y}^{2}+s \varphi_{y y}-t e^{k \varphi}, \quad \mu=b \varphi_{x}^{2}+a \varphi_{y}^{2}+s \varphi_{x x}-t e^{k \varphi} .
$$

The equation $[F, G]_{\mathcal{E}}=0$ simplify significantly in the case $a=1 / 4, s=-1 / 2$, which we consider. Studying compatibility of the arising scalar system $\mathcal{E} \subset J^{4}\left(\mathbb{R}^{2}\right)$ of $\operatorname{codim}(\mathcal{E})=3$ we get two family of solutions for the system (11).

The first corresponds to $b=1 / 2$ and a couple of the $1 \mathrm{D}$ Liouville equations: $\varphi_{x x}=\varphi_{y y}=-\frac{1}{2} e^{k \varphi}$.

The other possibility is $b=-1 / 2,(k, t)= \pm(2,1 / 2)$ and $\varphi$ satisfies the 2D Liouville equation $\varphi_{x x}+\varphi_{y y}=-e^{k \varphi} . \quad k=2$ is a particular case of the Jonas surfaces (related to projective transformations of minimal surfaces in $\mathbb{R}^{3}$ ).

In a similar way one can get Demoulin and many other known surfaces in $\mathbb{P}^{3}$ (see a relation of them to the soliton theory in $[\mathrm{F}]$ ).

2. Elliptic systems. The conformal quadric can be (locally) brought to the form $q_{\mathcal{E}}=d x^{2}+d y^{2}$. So the system $\mathcal{E}$ can be defined by the equations $F=r_{x x}-$ $r_{y y}+\phi\left(r_{x}, r_{y}, r\right), G=r_{x y}+\psi\left(r_{x}, r_{y}, r\right)$ with linear functions $\phi=a_{1} r_{x}+b_{1} r_{y}+c_{1} r$, $\psi=a_{2} r_{x}+b_{2} r_{y}+c_{2} r$.

The compatibility condition $[F, G]_{\mathcal{E}}=0$ consists of 4 equations on 6 functions, among which is the equation $\left(a_{1}+2 b_{2}\right)_{y}=\left(2 a_{2}-b_{1}\right)_{x}$. Then a rescaling $r \mapsto \rho \cdot r$ of the unknown function will simplify the equation to the form

$$
F=r_{x x}-r_{y y}+\alpha r_{x}+\beta r_{y}-\lambda r=0, G=2 r_{x y}+\beta r_{x}-\alpha r_{y}-\mu r=0 .
$$

Compatibility of this new $\mathcal{E}$ gives 3 equations on 4 functions $\alpha, \beta, \lambda, \mu$, which can be studied similarly to the hyperbolic case.

Let us also note that ansatzes of the hyperbolic case can be performed for the elliptic case as well: one takes the complex forms of the corresponding surfaces, to which the real surfaces are related by the real sections, and changes the section, getting (in the real context) elliptic surfaces etc.

Let us show to which PDEs the Kummer surface $K 3=\left\{z_{0}^{4}+z_{1}^{4}+z_{2}^{4}+z_{3}^{4}=0\right\}$ can be brought. A calculation gives the following elliptic system:

$$
\left\{\begin{array}{l}
x y^{3} r_{x x}+\left(1+y^{4}\right) r_{x y}=0 \\
x^{3} y r_{y y}+\left(1+x^{4}\right) r_{x y}=0
\end{array}\right.
$$

The corresponding Beltrami-Laplace equation simplifies to the following:

$$
L(u)=0, \quad L=x^{3} y^{3}\left[\left(x \partial_{x}+y \partial_{y}+1\right)^{2}-1\right]+x^{3}\left(y \partial_{y}^{2}-\partial_{y}\right)+y^{3}\left(x \partial_{x}^{2}-\partial_{x}\right) .
$$


Solving it we find the isothermal coordinates for the metric $q_{\mathcal{E}}$ :

$$
u=\operatorname{artan} x^{2}, \quad v=\ln \frac{y^{2}+\sqrt{1+x^{4}+y^{4}}}{\sqrt{1+x^{4}}} .
$$

Substituting $x=\sqrt{\tan u}, y=\sqrt{\sinh v / \cos u}$ into (14) we arrive at the system:

$$
r_{u u}-r_{v v}-(\csc 2 u-2 \cot 2 u) r_{u}-\operatorname{coth} 2 v \cdot r_{v}=0, \quad r_{u v}-\frac{1}{2} \tan u \cdot r_{v}=0 .
$$

Finally, with the change $r \mapsto r \cdot \sqrt[8]{4 / \cos ^{4} u \sin 2 u \sinh 2 v}$ we get the normal form

$$
\left\{\begin{aligned}
r_{u u}-r_{v v}+\frac{1}{2} \cot 2 u \cdot r_{u}-\frac{1}{2} \operatorname{coth} 2 v \cdot r_{v}+\left(\frac{9}{8}+\frac{5}{16}\left(\sin ^{-2} 2 u-\sinh ^{-2} 2 v\right)\right) r & =0 \\
2 r_{u v}-\frac{1}{2} \operatorname{coth} 2 v \cdot r_{u}-\frac{1}{2} \cot 2 u \cdot r_{v}+\frac{1}{8} \cot 2 u \operatorname{coth} 2 v \cdot r & =0 .
\end{aligned}\right.
$$

$\mathbf{2}^{+}$. Integration of Beltrami-Laplace equation. Let $(M, g)$ be a closed Riemannian surface such that the metric $g$ is geodesically equivalent to another metric $\bar{g}$, non-proportional to $g$ at least at one point (this implies, in particular, that the Euler characteristic of the surface is non-negative). Consider the Laplace operator $F=\Delta_{g}$. Then by a result of Matveev and Topalov ([MT $)$ there is a commuting independent second order differential operator $G$. It is an auxiliary integral, allowing us to integrate the Beltrami-Laplace equation globally. (The mentioned result holds also for $n$-dimensional Riemannian manifolds admitting non-proportional geodesically equivalent metrics.)

3. Parabolic systems. Pure parabolic systems are not of the form considered in Theorem 18. The canonical form of such systems is

$$
F=r_{x x}-\lambda r=0, G=r_{x y}+\alpha r_{x}+\beta r_{y}-\mu r=0, \quad \alpha, \beta, \lambda, \mu \in C^{\infty}\left(M^{2}\right) .
$$

In fact, transform the quadric to $q_{\mathcal{E}}=d y^{2}$. The system $\mathcal{E}$ is given, then by the equations $F=r_{x x}+\phi\left(r_{x}, r_{y}, r\right)=0, G=r_{x y}+\phi\left(r_{x}, r_{y}, r\right)$ with some linear $\phi, \psi$ as above. The system is characteristic and its compatibility condition is $\mathcal{D}_{x} \psi=\mathcal{D}_{y} \phi$. In particular, $b_{1}=0$ and the formula follows.

The case of mixed type equations, when a line of parabolic points separates the hyperbolic set on $\hat{M}^{2}$ from the elliptic one, is more interesting. Then Theorem 18 is applicable. The corresponding conformal metric $q_{\mathcal{E}}$ has several normal forms, each leading to a canonical form of the system $\mathcal{E}$.

4. Wilczynski tetrahedral. If a hyperbolic surface $\hat{M}^{2}$ is not ruled, $\beta^{2}+\gamma^{2} \neq 0$, the projectivized four points $R_{j}=\left[W_{j}(\mathbf{r})\right] \in \mathbb{P}^{3}, \mathbf{r}=\left(r_{0}, r_{1}, r_{2}, r_{3}\right)$, are well defined (do not depend on a choice of normalized coordinates $(x, y, r)$ ), where

$$
\begin{gathered}
W_{0}(r)=r, \quad W_{x}(r)=r_{x}-\frac{1}{2}(\ln |\gamma|)_{x} r, \quad W_{y}(r)=r_{y}-\frac{1}{2}(\ln |\beta|)_{y} r \\
W_{n}(r)=W_{x} \circ W_{y}(r)+\frac{1}{2}\left[(\ln |\beta|)_{x y}-\beta \gamma\right]=W_{y} \circ W_{x}(r)+\frac{1}{2}\left[(\ln |\gamma|)_{x y}-\beta \gamma\right] .
\end{gathered}
$$

The matrix $\left\|W_{j}\left(r_{i}\right)\right\|$ is non-degenerate, and so the points $R_{j}$ are not coplanar. They form the so-called Wilczynski moving tetrahedral ([W]). The vectors $R_{0} R_{x}$ and $R_{0} R_{y}$ are tangent to $\hat{M}^{2}$ at $R_{0}$ and $R_{0} R_{n}$ serves as a normal. Thus with every generic hyperbolic surface in $\mathbb{P}^{3}$ three other surfaces are canonically associated, which are diffeomorphic, but not necessarily projectively equivalent. 
For elliptic systems the above notions exist but only in the complexified space: one gets canonical but complex $R_{z}, R_{\bar{z}} \in \mathbb{C} P^{3}$ from

$$
W_{z}(r)=r_{z}-\frac{1}{2}(\ln \bar{a})_{z} r \text { and } W_{\bar{z}}(r)=r_{\bar{z}}-\frac{1}{2}(\ln a)_{\bar{z}} r, \quad a=\alpha+i \beta
$$

where as usual $\partial_{z}=\frac{1}{2}\left(\partial_{x}-i \partial_{y}\right), \partial_{\bar{z}}=\frac{1}{2}\left(\partial_{x}+i \partial_{y}\right)$. The point $R_{n}=\left[W_{n}(\mathbf{r})\right]$, however, is real, where

$$
W_{n}(r)=r_{x x}+r_{y y}-\phi \cdot r_{x}-\psi \cdot r_{y}+\left[\phi^{2}+\psi^{2}+2 \alpha^{2}+2 \beta^{2}\right],
$$

and $2 \phi=(\ln |a|)_{x}+(\arg a)_{y}, 2 \psi=(\ln |a|)_{y}-(\arg a)_{x}$. So in the elliptic case with $\alpha^{2}+\beta^{2} \neq 0$ there is a canonical normal also.

The above remarks, due to Theorem [18, give a possibility to multiply (equivalence classes of) pointwise generic non-degenerate compatible systems $\mathcal{E} \subset J^{2}\left(M^{2}\right)$ of codimension 2.

\section{REFERENCES}

[AB] M. Auslander, D. A. Buchsbaum, "Codimension and multiplicity", Annals of Math. 68, no. 3 (1958), 625-657. MR0099978 (20:6414)

[D] B. Dubrovin, "Geometry of 2D topological field theories", in: Springer LNM 1620 (1996), 120-348. MR1397274 (97d:58038)

[F] E. V. Ferapontov, "Integrable systems in projective differential geometry", Kyushu Jour. Math. 54, no. 1 (2000), 183-215. MR1762804(2001f:53030)

[Go] H. Goldschmidt, "Integrability criteria for systems of nonlinear partial differential equations", J. Diff. Geom., 1(3) (1967), 269-307. MR0226156 (37:1746)

[Gou] E. Goursat, "Lecons sur l'intégration des équations aux dérivées partielles du premier ordere", Hermann, Paris (1891).

[GR] H. Grauert, R. Remmert, "Analytische Stellenalgebren", Springer-Verlag (1971). MR0316742 (47:5290)

[Gu] V. Guillemin, "Some algebraic results concerning the characteristics of overdetermined partial differential equations", Amer. Journal of Math. 90 1968, 270-284. MR0223724 (36:6772)

[GS] V. Guillemin, S. Sternberg, "An algebraic model of transitive differential geometry", Bull. A.M.S., 70 (1964), 16-47. MR.0170295 (30:533)

[GQS] V. Guillemin, D. Quillen, S. Sternberg, "The integrability of characteristics", Comm. Pure Appl. Math. 23, no.1 (1970), 39-77. MR0461597(57:1582)

[Ha] R. Hartshorne, "Complete intersections and connectedness", Amer. Journ. Math. 84 (1962), 497-508. MR0142547 (26:116)

[Ho] L. Hörmander, "Linear partial differential operators", 3rd edition, Springer-Verlag (1969). MR0248435(40:1687)

[KLV] I. S. Krasilschik, V. V. Lychagin, A. M. Vinogradov, "Geometry of jet spaces and differential equations", Gordon and Breach (1986). MR0861121 (88m:58211)

[KL1] B.S. Kruglikov, V.V. Lychagin, "On equivalence of differential equations", Acta et Comment. Univ. Tartuensis Math. 3 (1999), 7-29. MR.1744128 (2000m:58001)

[KL2] B. S. Kruglikov, V. V. Lychagin, "Mayer brackets and solvability of PDEs - I", Diff. Geom. and its Appl. 17 (2002), 251-272. MR1925768 (2003f:35049)

[KS] A. Kumpera, D. Spencer, "Lie equations. Volume 1: General theory", Princeton University Press and University Tokyo Press (1972). MR0380908 (52:1805)

[LE] S. Lie, F. Engel, "Theorie der Transformationsgruppen", vol. II "Begründungstransformationen", Leipzig, Teubner (1888-1893).

[Ly] V.V. Lychagin, "Homogeneous geometric structures and homogeneous differential equations", in A. M. S. Transl., "The interplay between differential geometry and differential equations", V. Lychagin Eds., ser. 2, 167 (1995), 143-164. MR1343987 (96k:58249)

[Mum] D. Mumford, "Algebraic geometry I. Complex projective varieties", Springer-Verlag (1976). MR0453732 (56:11992)

[Man] Yu. I. Manin, "Frobenius manifolds, Quantum cohomology and Moduli spaces", A.M.S. Colloquium publ. 47 (1999). MR1702284(2001g:53156) 
[MT] V.S. Matveev, P. J. Topalov "Quantum integrability of Beltrami-Laplace operator as geodesic equivalence", Math. Z. 238 (2001), 833-866. MR1872577 (2002k:58068)

[S] D. C. Spencer, "Overdetermined systems of linear partial differential equations", Bull. Amer. Math. Soc., 75 (1969), 179-239. MR0242200 (39:3533)

[W] E. J. Wilczynski, "Projective differential geometry of curved surfaces I-V", Trans. Amer. Math. Soc., 8 (1907), 233-260; 9 (1908), 79-120, 293-315; 10 (1909), 176-200, 279-296. MR1500783 MR:1500804 MR1500815 MR1500833 MR:1500839

Institute of Mathematics and Statistics, University of Troms $\varnothing$, Troms $\varnothing$ 90-37, NorWAY

E-mail address: kruglikov@math.uit.no

Institute of Mathematics and Statistics, University of Troms $\varnothing$, Troms $\varnothing$ 90-37, NorWAY

E-mail address: lychagin@math.uit.no 